\title{
ALGEBRAIC $K$-THEORY AND TWISTED RECIPROCITY LAWS
}

\author{
BRUNO KAHN
}

\begin{abstract}
We extend the classical reciprocity laws of algebraic number theory to higher odd $K$-groups of algebraic integers. Moreover, we show that a suitable renormalisation of the corresponding "twisted Legendre symbols" is independent "in the average" from the chosen prime when the number field considered is Galois over Q. This seems new even in the classical case.
\end{abstract}

To Hyman Bass for his 70th birthday

\section{INTRODUCTION}

In this paper we generalise the classical reciprocity laws of algebraic number theory from $K_{1}$ (the units) to higher odd $K$-groups of rings of algebraic integers. Classically these laws concern "symbols" with values in roots of unity, while our symbols take their values in twisted roots of unity: for this reason we like to think of the laws governing them as twisted reciprocity laws.

Recall that, if $K$ is a number field, $\mathfrak{p}$ is a finite prime of $K$ and $a \in K^{*}$ is such that $v_{\mathfrak{p}}(a)=0$, the corresponding generalised Legendre symbol is defined by the formula

$$
\left(\frac{a}{\mathfrak{p}}\right)=f^{\frac{N(\mathfrak{p})-1}{m}}(\bmod \mathfrak{p})
$$

where $m$ is the number of roots of unity of $K$. In fact this is an $m$-th root of unity in the residue field $\kappa(\mathfrak{p})$, but it lifts canonically to an $m$-th root of unity of $K$ and is thought of as such. The generalised Legendre symbol is then extended into a generalised Jacobi symbol by

$$
\left(\frac{a}{\mathfrak{a}}\right)=\prod_{\mathfrak{p}}\left(\frac{a}{\mathfrak{p}}\right)^{v_{\mathfrak{p}}(\mathfrak{a})}
$$

provided that the fractional ideals $(a)$ and $\mathfrak{a}$ are coprime. A weak form of the reciprocity law (actually equivalent to the strong form) is that,

Date: 5-3-2003. 
for any $a$, there is a module $\mathfrak{m}$, i.e. a linear combination of primes of $K$ with nonnegative integer coefficients, such that

$$
\left(\frac{a}{(f)}\right)=1 \text { if } f \equiv 1\left(\bmod ^{*} \mathfrak{m}\right) .
$$

The most conceptual way to prove this formula is to interpret $\left(\frac{a}{\mathfrak{p}}\right)$ as the Hilbert symbol $(a, \pi)_{\mathfrak{p}}$, where $\pi$ is a uniformising parameter of the completion $K_{\mathfrak{p}}$. Hilbert symbols are now defined at all $\mathfrak{p}$ (including infinite places), not just those $\mathfrak{p}$ prime to $a$, and $\left(^{*}\right)$ follows from the Hilbert reciprocity law

$$
\prod_{\mathfrak{p}}(a, b)_{\mathfrak{p}}=1
$$

for any $a, b \in K^{*}$. This gives a more precise form of $\left(^{*}\right)$. An excellent and synthetic exposition of all this is given in the exercises of CasselsFröhlich [4].

We carry this over in the following way: we fix a rational prime number $l$. For $i>1$, we associate to each finite prime $\mathfrak{p} \nmid l$ a twisted Legendre symbol (see Definition 2.7 a))

$$
\left(\frac{-}{\mathfrak{p}}\right): K_{2 i-1}(K) \rightarrow H^{0}\left(K, \mathbf{Q}_{l} / \mathbf{Z}_{l}(i)\right)
$$

and prove a reciprocity law for them (Theorem 2.6). Actually, for $l=2$ we are obliged to assume that $K$ is totally imaginary for technical reasons (see Remarks 2.10 and 4.4). The proof is very parallel to the one in the classical context: we introduce twisted Hilbert symbols and prove a reciprocity formula for them (Theorem 4.3), from which Theorem 2.6 follows. In order to define the wild Hilbert symbols, i.e. those at places above $l$, we have to use Dwyer-Friedlander's étale $K$-theory: this makes our approach somewhat less elementary than one might hope at the outset. The twisted Hilbert reciprocity law then follows easily from (a small part of) the Poitou-Tate exact sequence.

The most intriguing result however comes when we modify the twisted Legendre symbols by changing their normalisation, i.e. multiplying them by certain integers prime to $l$ : we get variants $\left(\frac{-}{-}\right)^{(l)}$ (see Definition $2.7 \mathrm{~b})$ ). Then, in Theorem 2.8 , we show that $\left(\frac{-}{\mathfrak{p}}\right)^{(l)}$ is independent of $\mathfrak{p}$ "in the average", at least when $K$ is Galois over $\mathbf{Q}$. This seems new even in the classical case $i=1$. In the proof, the $l$-adic integer $\exp (l)(\exp (4)$ for $l=2)$ appears mysteriously... This contrasts 
starkly with the result of Arlettaz-Banaszak [1] which states that the map $K_{2 i-1}(K) \rightarrow \prod_{\mathfrak{p}} K_{2 i-1}(\kappa(\mathfrak{p}))$ has finite kernel. We don't know the exact significance of Theorem 2.8; Soule has suggested that it may have to do with the "field(s) with one element". Trying to prove it was motivated by [11] (see E.3 at the end of that paper, and Scholium 2.11 in the present one).

This paper is structured as follows. In Section 1 we give an easier, finite characteristic motivational analogue of our characteristic 0 results. In that case the results (Theorems 1.2 and 1.8) are much better, more general and easier to prove: they are closely related to Suslin's rigidity theorem [25]. In Section 2 we define the twisted Legendre and Jacobi symbols and their variants and state our main results, Theorems 2.6 and 2.8. The next sections up to Section 7 are devoted to the proofs. Finally, in Section 8, we give a computation of the twisted Hilbert symbol in terms of the Artin symbol, which connects our definition with a more classical approach of the Hilbert symbol.

We hope in the future to mix the results of Sections 1 and 2 to get twisted reciprocity laws for (at least some) smooth projective varieties over a number field.

Theorems 2.6 and 2.8 (the latter in the cyclotomic case) were found in the early nineties and explained in an Oberwolfach $K$-theory conference; Sections 1 and 8 are new.

I thank T. Nguyen Quang Do and M. Kolster for both recalling me that the generalised Moore reciprocity law of Theorem 4.3 is originally due to G. Banaszak [2], a fact I had embarrassingly forgotten in the preliminary version of this paper!

\section{The Situation In CHARACTERISTiC $p$}

We start by recalling Suslin's rigidity theorem [24]: let $k$ be an algebraically closed field and $X$ a smooth, projective, connected curve over $k$. Let $n$ be an integer prime to char $k$. Then, for any two closed points $x, y \in X$, the specialisation maps

$$
K_{*}(X, \mathbf{Z} / n) \underset{i_{y}^{*}}{\stackrel{i_{x}^{*}}{\rightrightarrows}} K_{*}(k, \mathbf{Z} / n)
$$

are equal.

Keep the same situation but relax the assumptions that $\operatorname{dim} X=1$ and $k$ is algebraically closed. For $x \in X_{(0)}$, we define the specialisation map at $x$

$$
s_{x}: K_{*}(X, \mathbf{Z} / n) \rightarrow K_{*}(k, \mathbf{Z} / n)
$$


as the composition

$$
K_{*}(X, \mathbf{Z} / n) \stackrel{i_{x}^{*}}{\longrightarrow} K_{*}(k(x), \mathbf{Z} / n) \stackrel{N_{k(x) / k}}{\longrightarrow} K_{*}(k, \mathbf{Z} / n) .
$$

These specialisation maps extend by linearity to a pairing

$$
Z_{0}(X) \times K_{*}(X, \mathbf{Z} / n) \rightarrow K_{*}(k, \mathbf{Z} / n) .
$$

By homotopy invariance of algebraic $K$-theory, this pairing factors as another pairing

$$
C H_{0}(X) \times K_{*}(X, \mathbf{Z} / n) \rightarrow K_{*}(k, \mathbf{Z} / n) .
$$

Recall that the proof of Suslin's theorem is just that, when $k$ is algebraically closed (and $\operatorname{dim} X=1$ ), (1.1) factors through the degree $\operatorname{map} \operatorname{Pic}(X) \rightarrow \mathbf{Z}$ because $\operatorname{Pic}^{0}(X)$ is divisible as the group of $k$-points of an abelian variety. The same argument, using [3, Lemma 1.4], gives:

1.1. Proposition. If $k$ is algebraically closed, the pairing (1.1) factors through the degree map deg : $\mathrm{CH}_{0}(X) \rightarrow \mathbf{Z}$.

Of course we have an analogous pairing without coefficients:

$$
C H_{0}(X) \times K_{*}(X) \rightarrow K_{*}(k) .
$$

1.2. Theorem. Suppose that $k$ is algebraic over $\mathbf{F}_{p}$ and that $X$ satisfies the Beilinson-Parshin conjecture: $K_{i}(X)$ is torsion for $i>0$ (e.g. $X$ is a curve, or an abelian variety on which the Tate conjecture holds, cf. [14]). Then (1.2) factors through the degree map.

Proof. Let $\bar{k}$ be an algebraic closure of $k$. Suppose first $*>0$. By Quillen [19], $K_{*}(k) \rightarrow K_{*}(\bar{k})$ is injective; hence we reduce to the case where $k=\bar{k}$. The argument is then the same as Suslin's.

For $*=0$, the theorem still holds, simply because $\operatorname{Ker}(\mathrm{deg})$ is torsion (Kato-Saito, [15]) and $K_{0}(k)=\mathbf{Z}$.

1.3. Remark. Assume that $X$ is geometrically connected, i.e. $k$ is its field of constants. Recall that $X$ has a 0 -cycle of degree 1 [23, Lemma 1 p. 325] Any such 0-cycle $z$ defines a retraction $s: K_{*}(X) \rightarrow K_{*}(k)$ of the map $K_{*}(k) \rightarrow K_{*}(X)$. A reformulation of Theorem 1.2 is that $s$ does not depend on $z$ and that, for any $x \in X_{(0)}$, one has

$$
s_{x}=\operatorname{deg}(x) s .
$$

From now on we assume that $X$ is geometrically connected, as in Remark 1.3. We are going to prove a similar but more bizarre theorem. First: 
1.4. Lemma. Let $\mathbf{F}_{q}$ be a finite field and $n \geq 1, i \geq 1$ two integers. Then the composition

$$
K_{2 i-1}\left(\mathbf{F}_{q^{n}}\right) \stackrel{N}{\longrightarrow} K_{2 i-1}\left(\mathbf{F}_{q}\right) \stackrel{\iota}{\longleftrightarrow} K_{2 i-1}\left(\mathbf{F}_{q^{n}}\right)
$$

equals multiplication by $\frac{q^{n i}-1}{q^{i}-1}=\frac{\left|K_{2 i-1}\left(\mathbf{F}_{q^{n}}\right)\right|}{\left|K_{2 i-1}\left(\mathbf{F}_{q}\right)\right|}$.

Proof. By Quillen [19], $K_{2 i-1}\left(\mathbf{F}_{q}\right) \rightarrow K_{2 i-1}\left(\mathbf{F}_{q^{n}}\right)$ is injective and Frobenius acts on $K_{2 i-1}\left(\mathbf{F}_{q^{n}}\right)$ by multiplication by $q^{i}$.

1.5. Lemma. Let $l$ be a prime number and $u \in \mathbf{C}_{l}$ be such that $v\left(\frac{u-1}{2}\right)>0$, where $v$ is the valuation of $\mathbf{C}_{l}$. Then, for any integer $n>0$, we have

$$
\frac{u^{n}-1}{n(u-1)} \equiv 1 \quad\left(\bmod \frac{u-1}{2} \mathbf{O}_{l}\right)
$$

where $\mathbf{O}_{l}$ is the valuation ring of $\mathbf{C}_{l}$. In particular, $v\left(\frac{u^{n}-1}{u-1}\right)=v(n)$.

Proof. Let $a=u-1$ and $b=u^{s}-1$. The identity

$$
\frac{(1+a)^{s t}-1}{s t a}=\frac{(1+b)^{t}-1}{t b} \cdot \frac{(1+a)^{s}-1}{s a}
$$

and an induction on the number of prime factors of $n$ reduces us to two cases:

- $(n, l)=1$;

- $n=l$.

In the first case, we have

$$
\frac{(1+a)^{n}-1}{a}=n+\left(\begin{array}{l}
n \\
2
\end{array}\right) a+\cdots \equiv n \quad\left(\bmod \frac{a}{2} \mathbf{O}_{l}\right)
$$

hence the claim.

In the second case, we have

$$
\frac{(1+a)^{l}-1}{l a}=1+(l-1) \frac{a}{2}+\cdots \equiv 1 \quad\left(\bmod \frac{a}{2} \mathbf{O}_{l}\right)
$$

again.

1.6. Corollary. With notation as in Lemma 1.4, we have for any $x \in$ $K_{2 i-1}\left(\mathbf{F}_{q^{n}}\right)$ :

$$
2(\iota N(x)-n x)=0 .
$$

This follows from Lemmas 1.4 and 1.5. 
1.7. Definition. Let $l$ be a prime number and $x \in X_{(0)}$.

a) We write

$$
\operatorname{deg}_{l}(x)=|\operatorname{deg}(x)|_{l}^{-1}
$$

where $|\cdot|_{l}$ denotes the normalised $l$-adic absolute value (it is just the $l$-part of $\operatorname{deg}(x))$.

b) We define

$$
s_{x}^{(l)}: K_{2 i-1}(X) \rightarrow K_{2 i-1}(k(x))\{l\}
$$

by

$$
s_{x}^{(l)}(a)=\operatorname{deg}_{l}(x) i_{x}^{*} a .
$$

Note that, by Corollary 1.6, we have

$$
2\left(\iota s_{x}(a)-\operatorname{deg}(x) i_{x}^{*}(a)\right)=0 .
$$

Hence, from Theorem 1.2:

1.8. Theorem. a) If $l \neq 2$, then for any $x \in X$ :

$$
s_{x}^{(l)}=\operatorname{deg}_{l}(x) s
$$

where $s$ is as in Remark 1.3.

b) If $l=2$, then then for any $x \in X$ :

$$
s_{x}^{(l)}=\operatorname{deg}_{l}(x) s+s^{\prime}
$$

where $s$ is as in Remark 1.3 and $2 s^{\prime}=0$.

In particular $s_{x}^{(l)}(a) \in K_{2 i-1}(k)\{l\}$ for any $a$, and it does not depend on $x$ for $\operatorname{deg}_{l}(x)=1$.

1.9. Remark. Suppose $X$ is a curve. According to the (still unproven!) Quillen-Lichtenbaum conjecture, the natural map

$$
K_{2 i-1}(k) \otimes K_{0}(X) \rightarrow K_{2 i-1}(X)
$$

should induce an isomorphism

$$
K_{2 i-1}(k)\{l\} \oplus K_{2 i-1}(k)\{l\} \stackrel{\sim}{\longrightarrow} K_{2 i-1}(X)\{l\}
$$

for any $l \neq$ char $k$, via the canonical isomorphism $K_{0}(X)=\mathbf{Z} \oplus \operatorname{Pic}(X)$ and the degree map $\operatorname{deg}: \operatorname{Pic}(X) \rightarrow \mathbf{Z}$. At least, it is immediate to check that for a point $x \in X$, the composition of $s_{x}$ (resp. $\left.s_{x}^{(l)}\right)$ with this homomorphism is $(\operatorname{deg}(x), 0)$ (resp. $\left.\left(\operatorname{deg}_{l}(x), 0\right)\right)$. Therefore, Theorems 1.2 and 1.8 are not particularly interesting in this case once the conjecture is proven. Nevertheless we have given full proofs as a motivation for the sequel of this article, the number field case. We shall get an analogue to (1.2) (Theorem 2.6) and a partial analogue to Theorem 1.8 (Theorem 2.8). 


\section{The Number FIELD CASE}

From now on, we fix a prime number $l$. If $F$ is a field of characteristic $\neq l$ and $i>0$, we write

$$
m_{i}(F)=\left|H^{0}\left(F, \mathbf{Q}_{l} / \mathbf{Z}_{l}(i)\right)\right| .
$$

Then $m_{i}(F)$ is finite in the following cases:

- $F$ is finitely generated.

- $F$ is a $p$-adic field.

- $F=\mathbf{R}$ and $i$ is odd; then $m_{i}(F)= \begin{cases}1 & \text { if } l \neq 2 \\ 2 & \text { if } l=2 .\end{cases}$

Suppose first that $F$ is finite with $q$ elements. It follows from [19] that one has isomorphisms

$$
K_{2 i-1}(F)\{l\} \simeq H^{0}\left(F, \mathbf{Q}_{l} / \mathbf{Z}_{l}(i)\right) .
$$

For the sequel, we need to fix such isomorphisms explicitly. Quillen's theorem implies that the map

$$
K_{2 i-1}(F) \rightarrow H^{0}\left(F, K_{2 i-1}(\bar{F})\right)
$$

is an isomorphism, where $\bar{F}$ is an algebraic closure of $F$. Therefore it suffices to produce an explicit isomorphism $K_{2 i-1}(\bar{F})\{l\} \stackrel{\sim}{\rightarrow} \mathbf{Q}_{l} / \mathbf{Z}_{l}(i)$.

If $(n, q)=1$, we have an isomorphism

$$
K_{2 i}(\bar{F}, \mathbf{Z} / n) \stackrel{\sim}{\longrightarrow}{ }_{n} K_{2 i-1}(\bar{F})
$$

since $K_{2 i}(\bar{F})=0$. For $i=1$, this gives an isomorphism

$$
K_{2}(\bar{F}, \mathbf{Z} / n) \stackrel{\sim}{\longrightarrow} \mu_{n}=\mathbf{Z} / n(1) .
$$

On the other hand, the product in $K$-theory with coefficients gives an isomorphism (take $n$ large enough to avoid nonexistent problems with the product...)

$$
K_{2}(\bar{F}, \mathbf{Z} / n)^{\otimes i} \stackrel{\sim}{\longrightarrow} K_{2 i}(\bar{F}, \mathbf{Z} / n) .
$$

Putting this together, we get isomorphisms for all $n$ prime to $q$

$$
{ }_{n} K_{2 i-1}(\bar{F}) \stackrel{\sim}{\longrightarrow} / n(i)
$$

hence the desired isomorphisms in the limit, since the groups $K_{2 i-1}(\bar{k})$ are torsion prime to $q$. These isomorphisms obviously commute with extension and restriction of scalars.

Let now $F$ be a finite extension of $\mathbf{Q}_{p}(p \neq l), O_{F}$ its valuation ring and $k$ its residue field. There are canonical maps

$$
K_{*}\left(O_{F}\right) \rightarrow K_{*}(k)
$$


hence a canonical map

$$
i_{F}^{*}: K_{2 i-1}\left(O_{F}\right) \rightarrow H^{0}\left(k, \mathbf{Q}_{l} / \mathbf{Z}_{l}(i)\right)
$$

defined as the composition of (2.3), the projection of $K_{2 i-1}(k)$ onto its $l$-primary component and the explicit isomorphism (2.2) described above.

Let finally $K$ be a number field and $O_{K}$ its ring of integers. Throughout the paper we write

$$
R_{K}:=O_{K}[1 / l]
$$

Let $\mathfrak{p}$ a prime of $R_{K}$. Composing $i_{K_{\mathfrak{p}}}$ with the canonical map $K_{2 i-1}\left(R_{K}\right) \rightarrow K_{2 i-1}\left(O_{K_{\mathfrak{p}}}\right)$, we get a canonical map

$$
i_{\mathfrak{p}}^{*}: K_{2 i-1}\left(R_{K}\right) \rightarrow H^{0}\left(\kappa(\mathfrak{p}), \mathbf{Q}_{l} / \mathbf{Z}_{l}(i)\right) .
$$

On the other hand, there is a canonical injection

$$
\iota: H^{0}\left(K, \mathbf{Q}_{l} / \mathbf{Z}_{l}(i)\right)(\stackrel{\sim}{\leftarrow})^{-1} H^{0}\left(R_{K}, \mathbf{Q}_{l} / \mathbf{Z}_{l}(i)\right) \hookrightarrow H^{0}\left(\kappa(\mathfrak{p}), \mathbf{Q}_{l} / \mathbf{Z}_{l}(i)\right)
$$

that we use to identify $H^{0}\left(K, \mathbf{Q}_{l} / \mathbf{Z}_{l}(i)\right)$ to a subgroup of $H^{0}\left(\kappa(\mathfrak{p}), \mathbf{Q}_{l} / \mathbf{Z}_{l}(i)\right)$.

2.1. Lemma. Let $m_{i}=m_{i}(K)$ and $m_{i}(\mathfrak{p})=m_{i}(\kappa(\mathfrak{p}))$. Then, if $v_{l}\left(m_{i} / 2\right)>0$, the quotient $\frac{m_{i}(\mathfrak{p})}{m_{i}}$ does not depend on the choice of $i$. This happens as soon as $l-1 \mid i(l$ odd) or $2 \mid i(l=2)$.

Proof. Let $G_{K}$ be the absolute Galois group of $K, G_{l}$ its maximal $l$ ramified quotient, $G_{\mathfrak{p}}$ the absolute Galois group of $\kappa(\mathfrak{p})$ and $\kappa: G_{K} \rightarrow$ $\mathbf{Z}_{l}^{*}$ the cyclotomic character. Since the maximal $l$-cyclotomic extension of $K$ is $l$-ramified, $\kappa$ factors through $G_{l}$. Let $\Gamma_{K}=\kappa\left(G_{K}\right)=\kappa\left(G_{l}\right) \subseteq \mathbf{Z}_{l}^{*}$ and $\Gamma_{\mathfrak{p}}=\kappa\left(G_{\mathfrak{p}}\right)$. Then $\Gamma_{\mathfrak{p}}$ is a subgroup of finite index of $\Gamma_{K}$.

If $l>2$, we may write

$$
\begin{aligned}
\Gamma_{K} & =\mu_{d} \times\left(1+l^{n} \mathbf{Z}_{l}\right) \\
\Gamma_{\mathfrak{p}} & =\mu_{d_{\mathfrak{p}}} \times\left(1+l^{n_{\mathfrak{p}}} \mathbf{Z}_{l}\right)
\end{aligned}
$$

with $d, d_{\mathfrak{p}}$ prime to $l$. If $l=2$, we may write

$$
\begin{aligned}
\Gamma_{K}^{2} & =1+2^{n+1} \mathbf{Z}_{l} \\
\Gamma_{\mathfrak{p}}^{2} & =1+2^{n_{\mathfrak{p}}+1} \mathbf{Z}_{l}
\end{aligned}
$$

with $2 \leq n \leq n_{\mathfrak{p}}$ 
For $l>2$, a simple computation shows that $m_{i} \neq 1 \Longleftrightarrow d \mid i$ (resp. $\left.m_{i}(\mathfrak{p}) \neq 1 \Longleftrightarrow d_{\mathfrak{p}} \mid i\right)$, and in these cases

$$
\begin{aligned}
m_{i} & =l^{n+v_{l}(i)} \\
m_{i}(\mathfrak{p}) & =l^{n_{\mathfrak{p}}+v_{l}(i)}
\end{aligned}
$$

hence

$$
\frac{m_{i}(\mathfrak{p})}{m_{i}}=l^{n_{\mathfrak{p}}-n} .
$$

The reasoning for $l=2$ is similar.

2.2. Definition. For $\mathfrak{p}$ prime to $l$, we denote the integer of Lemma 2.1 by $\operatorname{deg}_{l}(\mathfrak{p})$. For a fractional ideal $\mathfrak{a}$ prime to $l$, we define

$$
\operatorname{deg}_{l}(\mathfrak{a})=\sum_{\mathfrak{p} \nmid l} v_{\mathfrak{p}}(\mathfrak{a}) \operatorname{deg}_{l}(\mathfrak{p}) .
$$

2.3. Remarks. 1) Keep the notation in the proof of Lemma 2.1. Then $q=N(\mathfrak{p})$ generates $\Gamma_{\mathfrak{p}}$. Hence for $l$ odd we may compute $d_{\mathfrak{p}}, n_{\mathfrak{p}}$ as follows:

$$
\begin{aligned}
& d_{\mathfrak{p}}=\text { order of } q \text { in }(\mathbf{Z} / l)^{*} \\
& n_{\mathfrak{p}}=v_{l}\left(q^{d_{\mathfrak{p}}}-1\right)=v_{l}\left(q^{l-1}-1\right)
\end{aligned}
$$

while for $l=2$

$$
n_{\mathfrak{p}}=v_{2}\left(q^{2}-1\right)-1
$$

2) It is probably not true that for a given $\mathfrak{p}$, the set of primes $l$ such that $\operatorname{deg}_{l}(\mathfrak{p}) \neq 1$ is finite, but at least this set seems "small". For example, if $K=\mathbf{Q}$ and $p \neq l$ is a rational prime, then (assuming $l$ odd), with notation as in the proof of Lemma 2.1, we have $d=l-1$, $n=1$ (Gauß). Taking the previous remark into account, this shows that $\operatorname{deg}_{l}(p) \neq 1$ if and only if

$$
p^{l-1} \equiv 1 \quad\left(\bmod l^{2}\right) .
$$

This condition appears in proofs of the first case of Fermat's Last Theorem by Wieferich et al. in the early 20th century; it has been checked numerically that it occurs very rarely. A heuristic argument shows that the number of such $l \leq x$ should be asymptotic to $\log \log x$.

On the other hand, for a given $l$, the set of $\mathfrak{p}$ such that $\operatorname{deg}_{l}(\mathfrak{p}) \neq 1$ has a density $(\in] 0,1[$ except when $l=2$ and $K$ is exceptional) by Čbotarev's density theorem, cf. [8]. 
2.4. Definition. a) Let $\mathfrak{p} \nmid l$. Let $m_{i}=m_{i}(K)$ and $N(\mathfrak{p})$ the norm of $\mathfrak{p}$ (the cardinality of the residue field). For $a \in K_{2 i-1}\left(R_{K}\right)$, we set

$$
s_{\mathfrak{p}}(a)=\left(\frac{a}{\mathfrak{p}}\right)=\iota^{-1}\left(\frac{N(\mathfrak{p})^{i}-1}{m_{i}} i_{\mathfrak{p}}^{*}(a)\right) \in H^{0}\left(K, \mathbf{Q}_{l} / \mathbf{Z}_{l}(i)\right)
$$

where $i_{\mathfrak{p}}^{*}($ resp. ८) is the map of $(2.4)$ (resp. (2.6)).

b) Let $\mathfrak{a}$ be a fractional ideal prime to $l$. We set

$$
\left(\frac{a}{\mathfrak{a}}\right)=\sum_{\mathfrak{p}} v_{\mathfrak{p}}(\mathfrak{a})\left(\frac{a}{\mathfrak{p}}\right) .
$$

These are the twisted Legendre symbol and the twisted Jacobi symbol.

2.5. Example. For $i=1$ we recover the classical generalised Legendre and Jacobi symbols (with an additive notation).

2.6. Theorem. Suppose that either $l$ is odd or $K$ is totally imaginary.Then there is a modulus $\mathfrak{m}_{i}$ with support on prime divisors of $l$ such that, for all $a \in K_{2 i-1}\left(R_{K}\right),\left(\frac{a}{(f)}\right)=0$ for any $f \in K^{*}$ such that $f \equiv 1\left(\bmod ^{*} \mathfrak{m}_{i}\right)$

2.7. Definition. a) For a prime $\mathfrak{p} \nmid l$ and $a \in K_{2 i-1}\left(R_{K}\right)$, we set

$$
s_{\mathfrak{p}}^{(l)}(a)=\left(\frac{a}{\mathfrak{p}}\right)^{(l)}=\operatorname{deg}_{l}(\mathfrak{p}) i_{\mathfrak{p}}^{*}(a) \in H^{0}\left(K, \mathbf{Q}_{l} / \mathbf{Z}_{l}(i)\right)
$$

so that we have

$$
s_{\mathfrak{p}}(a)=\frac{N(\mathfrak{p})^{i}-1}{m_{i}(\mathfrak{p})} s_{\mathfrak{p}}^{(l)}(a) .
$$

b) Let $\mathfrak{a}$ be a fractional ideal prime to $l$. We set

$$
\left(\frac{a}{\mathfrak{a}}\right)^{(l)}=\sum_{\mathfrak{p}} v_{\mathfrak{p}}(\mathfrak{a})\left(\frac{a}{\mathfrak{p}}\right)^{(l)}
$$

Note that working with $s_{\mathfrak{p}}(a)$ or $s_{\mathfrak{p}}^{(l)}(a)$ is equivalent in the sense that $\frac{N(\mathfrak{p})^{i}-1}{m_{i}(\mathfrak{p})}$ is prime to $l$. The same is not true for $\left(\frac{a}{\mathfrak{a}}\right)$ and $\left(\frac{a}{\mathfrak{a}}\right)^{(l)}$ when $\mathfrak{a}$ is not prime.

2.8. Theorem. Let $K$ be a Galois extension of $\mathbf{Q}$ and $K_{0}$ the maximal $l$-cyclotomic subextension of $K$. Then

a) If $l$ is odd, there exists a homomorphism $s: K_{2 i-1}\left(R_{K}\right) \rightarrow$ $H^{0}\left(K, \mathbf{Q}_{l} / \mathbf{Z}_{l}(i)\right)$ such that, for any rational number $r$ prime to $l$, one has identically

$$
\left(\frac{a}{\left(r R_{K}\right)}\right)^{(l)}=\frac{\operatorname{deg}_{l}\left(r R_{K}\right)}{\left[K: K_{0}\right]} s(a)
$$


b) If $l=2$ and $K_{0}$ is totally imaginary, there exist two homomorphisms $s_{1}, s_{2}: K_{2 i-1}\left(R_{K}\right) \rightarrow H^{0}\left(K, \mathbf{Q}_{2} / \mathbf{Z}_{2}(i)\right)$, with $2 s_{1}=0$, such that, for any rational number $r$ with $v_{2}(r)=0$, one has identically

$$
\left(\frac{a}{r R_{K}}\right)^{(2)}=\frac{r-1}{2} s_{1}(a)-\frac{\operatorname{deg}_{2}\left(r R_{K}\right)}{2\left[K: K_{0}\right]} s_{2}(a) .
$$

(In the course of the proof it will be shown that $\frac{\operatorname{deg}_{l}\left(r R_{K}\right)}{2\left[K: K_{0}\right]}$ is an $l$-integer for all $l$, which gives a meaning to the formulas.)

2.9. Corollary. In Theorem 2.8, assume that $K=K_{0}$ and that $r$ is a prime number $p$. Let $n=v_{l}([K: \mathbf{Q}])+1$.

a) If $l$ is odd, $n \geq 2$ and $p$ is inert in Theorem 2.8, then $s_{p}^{(l)}(a)$ does not depend on $p$.

b) If $l=2, n \geq 3$ and $p \equiv \pm 5(\bmod 8)$ in Theorem 2.8, then $s_{p}^{(l)}(a)$ only depends on the classical Legendre symbol $\left(\frac{-1}{p}\right)$.

Indeed, in a), $p$ inert means that $p R_{K}$ is prime and the hypothesis $n \geq 2$ implies that $\operatorname{deg}_{l}\left(p R_{K}\right)=1$. Similarly, in b), $n \geq 3$ implies $\operatorname{deg}_{2}\left(p R_{K}\right)=2$.

Theorem 2.6 will be proven in Section 5 as a corollary of a reciprocity formula (Theorem 5.5). Theorem 2.8 will be proven in Section 7 ; moreover, explicit values of $s, s_{1}$ and $s_{2}$ will be given in the proof.

2.10. Remark. Theorems 2.6, 2.8 and 5.5 are consequences of Theorem 4.3 which states that a certain sequence is a complex. The only reason why the formally real case is excluded from the first three theorems for $l=2$ is that we cannot prove Theorem 4.3 in this case. (See Remark 4.4 in this respect.) Granting it, let us indicate what the statements of Theorem 2.6 and 2.8 become in the real case:

- Theorem 2.6 remains the same for $i \not \equiv 1(\bmod 4)$. For $i \equiv 1$ (mod 4$)$, the condition on $f$ must be strengthened by requiring that $f$ is totally positive.

- In the formula of Theorem $2.8 \mathrm{~b}$ ), two changes occur: the factor $\frac{\operatorname{deg}_{2}\left(r R_{K}\right)}{2\left[K: K_{0}\right]}$ must be replaced by $\frac{\operatorname{deg}_{2}\left(r R_{K}\right)}{\left[K: K_{0}\right]}$ (this is explained by formulas $(7.1))$ and when $i \equiv 1(\bmod 4)$ a third summand of the form $\operatorname{sgn}(r) s_{3}(a)$ appears, where $\operatorname{sgn}(r)$ is the sign of $r$ (viewed in $\mathbf{Z} / 2$ ) and $2 s_{3}=0$. 
2.11. Scholium. It follows from the work of Harris-Segal [8] and DwyerFriedlander-Mitchell [6] that, for $l$ odd, there are canonical homomorphisms

$$
\beta^{i}: H^{0}\left(K, \mathbf{Q}_{l} / \mathbf{Z}_{l}(i)\right) \rightarrow K_{2 i-1}\left(R_{K}\right) .
$$

This is still true if $l=2$ and $\sqrt{-1} \in K$. (See [11] for a homotopical strengthening of this result and [7] for an extension to the case $l=$ 2, $K$ non-exceptional.) These homomorphisms are number-theoretic analogues of the homomorphisms $K_{*}(k) \rightarrow K_{*}(X)$ where $X$ is a smooth curve over a field $k$, as in Section 1. By its very construction, $\beta^{i}$ is a retraction of $s_{\mathfrak{p}}^{(l)}$ when $\mathfrak{p}$ is a prime of $K$ such that $\kappa(\mathfrak{p})$ has the "same cyclotomy" as $K$, i.e. $\operatorname{deg}_{l}(\mathfrak{p})=1$. In general, one checks easily that

$$
s_{\mathfrak{p}}^{(l)} \circ \beta^{i}=\operatorname{deg}_{l}(\mathfrak{p}) .
$$

Theorem 2.8 says that $s_{\mathfrak{p}}$ is independent of $\mathfrak{p}$ "in the average", at least for Galois extensions $K$ of $\mathbf{Q}$ (strictly speaking, this interpretation is correct only when $K$ is cyclotomic). In this sense it is a weak analogue of Theorem 1.8.

\section{COMPARING PAIRINGS}

For a topological $\mathbf{Z}_{l}^{*}$-module $A$ and $i \in \mathbf{Z}$, we denote by $A(i)$ the topological $\mathbf{Z}_{l}^{*}$-module with new action $\rho^{i}$ given by $\rho^{i}(u) a=u^{i} \rho(u) a$, where $\rho$ is the original action.

Let $i \neq 0$, and let $\Gamma$ be an open subgroup of $\mathbf{Z}_{l}^{*}$. The groups $H^{0}\left(\Gamma, \mathbf{Q}_{l} / \mathbf{Z}_{l}(i)\right)$ and $H^{0}\left(\Gamma, \mathbf{Q}_{l} / \mathbf{Z}_{l}(-i)\right)$ are then finite and cyclic of the same order. Let $m$ be an integer dividing this order. We have a perfect duality of finite groups

$$
\alpha_{m}: H^{0}(\Gamma, \mathbf{Z} / m(-i)) \times H^{0}(\Gamma, \mathbf{Z} / m(i)) \rightarrow H^{0}(\Gamma, \mathbf{Z} / m)=\mathbf{Z} / m
$$

These pairings are related as follows:

3.1. Lemma. Let $m \mid m^{\prime}$ dividing the order of $H^{0}\left(\Gamma, \mathbf{Q}_{l} / \mathbf{Z}_{l}(i)\right)$. Then, under the pairings $\alpha_{m}$ and $\alpha_{m^{\prime}}$, the transpose of the inclusion map $H^{0}(\Gamma, \mathbf{Z} / m(i)) \hookrightarrow H^{0}\left(\Gamma, \mathbf{Z} / m^{\prime}(i)\right)$ is multiplication by $m^{\prime} / m$.

3.2. Proposition. Let $\chi_{i}: \Gamma \rightarrow \mathbf{Z} / m$ be the character given by the formula

$$
\chi_{i}(\gamma)=\frac{\gamma^{i}-1}{m} \quad(\bmod m)
$$

where $m$ divides $\left|H^{0}\left(\Gamma, \mathbf{Q}_{l} / \mathbf{Z}_{l}(i)\right)\right|$. Then the Bockstein homomorphism $\beta: H^{0}(\Gamma, \mathbf{Z} / m(i)) \rightarrow H^{1}(\Gamma, \mathbf{Z} / m(i))$ associated to the exact sequence of $\Gamma$-modules

$$
0 \rightarrow \mathbf{Z} / m(i) \rightarrow \mathbf{Z} / m^{2}(i) \rightarrow \mathbf{Z} / m(i) \rightarrow 0
$$


equals cup-product by $\chi_{i}$.

Proof. Let $x \in H^{0}(\Gamma, \mathbf{Z} / m(i))$. A 1-cocycle $c$ representing $\beta x$ may be described as follows: lift $x$ to $\tilde{x} \in \mathbf{Z} / m^{2}(i)$. For $\gamma \in \Gamma$, we then have $(\gamma-1) \tilde{x}=m c(\gamma)$ for a well-defined $c(\gamma) \in \mathbf{Z} / m(i)=m \mathbf{Z} / m^{2}(i)$. Proposition 3.2 follows.

Let now $F$ be a finite extension of $\mathbf{Q}_{p}$, with $p \neq l$. We denote by $k$ its residue field, and let $q:=|k|$. For $m \mid m_{i}(F)$ (see (2.1)), we have a composite isomorphism $\theta_{m}$ :

$$
H^{0}(F, \mathbf{Z} / m(i)) \stackrel{\sim}{\longrightarrow} H^{0}(F, \mathbf{Z} / m(-i))^{*} \stackrel{\sim}{\longrightarrow} H^{2}(F, \mathbf{Z} / m(i+1))
$$

where the first isomorphism stems from (3.1) and the second one comes from local Tate duality.

3.3. Remark (compare [18, Remark 15.9]). The isomorphism $\theta_{m}$ may also be described as cup-product by $e_{m}$, where $e_{m} \in{ }_{m} \operatorname{Br}(F)=$ $H^{2}(F, \mathbf{Z} / m(1))$ is the generator such that $\operatorname{inv}\left(e_{m}\right)=1 \in \mathbf{Z} / m$, where inv $:{ }_{m} \operatorname{Br}(F) \rightarrow \mathbf{Z} / m$ is the isomorphism stemming from class field theory, with the normalisation that Frobenius elements correspond to uniformising parameters. This is obvious.

Finally, we have a residue isomorphism

$$
\partial: H^{2}(F, \mathbf{Z} / m(i+1)) \stackrel{\sim}{\longrightarrow} H^{1}(k, \mathbf{Z} / m(i))
$$

a specialisation isomorphism

$$
s: H^{0}(F, \mathbf{Z} / m(i)) \stackrel{\sim}{\longrightarrow} H^{0}(k, \mathbf{Z} / m(i))
$$

and a Bockstein isomorphism

$$
\beta: H^{0}(k, \mathbf{Z} / m(i)) \stackrel{\sim}{\longrightarrow} H^{1}(k, \mathbf{Z} / m(i))
$$

already considered in Proposition 3.2.

3.4. Proposition. For $l \neq p$, the following diagram commutes:

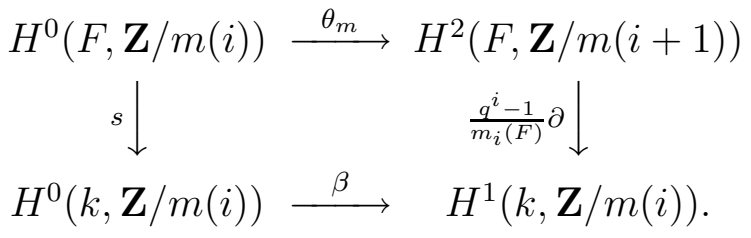

Proof. We have a commutative diagram of pairings

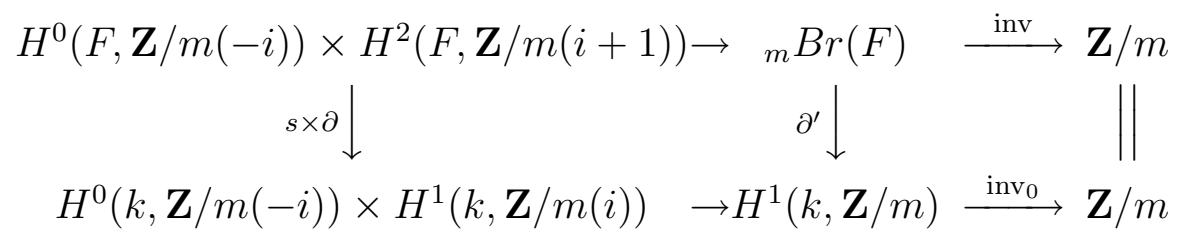


where $\operatorname{inv}_{0}$ carries a character $\chi$ to $\chi($ Frob $)$, where Frob is the arithmetic Frobenius. The result follows from this and Proposition 3.2, since the cyclotomic character of Frob is $q$.

\section{Generalised Moore reciprocity}

We shall use Dwyer-Friedlander's étale $K$-theory [5]. Recall that, for any $\mathbf{Z}[1 / l]$-scheme $X$ of finite étale $l$-cohomological dimension, there are groups $K_{*}^{\text {ét }}(X)$, maps $K_{*}(X) \rightarrow K_{*}^{\text {ét }}(X)$ and a spectral sequence [5, Prop. 5.1]

$$
E_{2}^{p, q}=H_{\text {ét }}^{p}\left(X, \mathbf{Z}_{l}(-q / 2)\right) \Rightarrow K_{-p-q}^{\text {ét }}(X)
$$

where the $E_{2}$-terms are continuous étale cohomology groups ${ }^{1}$.

If $k$ is a finite field, then the Dwyer-Friedlander spectral sequence degenerates into isomorphisms

$$
K_{2 i-1}^{\text {ét }}(k) \stackrel{\sim}{\longrightarrow} H^{1}\left(k, \mathbf{Z}_{l}(i)\right) \quad(i \in \mathbf{Z}) .
$$

4.1. Lemma. For $i>0$, let $\rho_{1}: K_{2 i-1}(k) \rightarrow H^{0}\left(k, \mathbf{Q}_{l} / \mathbf{Z}_{l}(i)\right)$ be the homomorphism from (2.2) and $\rho_{2}$ the composite homomorphism $K_{2 i-1}(k) \rightarrow K_{2 i-1}^{\text {ét }}(k) \rightarrow H^{1}\left(k, \mathbf{Z}_{l}(i)\right)$. Then the diagram

$$
\begin{aligned}
& H^{0}\left(k, \mathbf{Q}_{l} / \mathbf{Z}_{l}(i)\right) \\
& \rho_{1} \nearrow \\
& K_{2 i-1}(k) \quad \beta \downarrow \\
& \rho_{2} \searrow \\
& H^{1}\left(k, \mathbf{Z}_{l}(i)\right)
\end{aligned}
$$

commutes, where $\beta$ is the Bockstein homomorphism from the exact sequence

$$
0 \rightarrow \mathbf{Z}_{l}(i) \rightarrow \mathbf{Q}_{l}(i) \rightarrow \mathbf{Q}_{l} / \mathbf{Z}_{l}(i) \rightarrow 0
$$

Proof. The Dwyer-Friedlander spectral sequence may be interpreted as the descent spectral sequence associated to the weak equivalence

$$
K^{\text {ét }}(k) \stackrel{\sim}{\longrightarrow} \mathbb{H}_{\text {ét }}\left(k, K^{\text {ét }}\right)
$$

where we use Thomason's hypercohomological notation. We may smash this weak equivalence with another spectrum and get a new spectral sequence. Smashing it in particular with the homotopy fibre sequence

$$
\mathbb{S} \rightarrow M(\mathbf{Z}[1 / l]) \rightarrow M\left(\mathbf{Q}_{l} / \mathbf{Z}_{l}\right) \rightarrow \Sigma \mathbb{S}
$$

\footnotetext{
${ }^{1}$ To avoid fringe effects we work with the non-connective version of DwyerFriedlander's étale $K$-theory, although this makes in fact no difference in the cases we'll consider.
} 
where $\mathbb{S}$ is the sphere spectrum and $M(\mathbf{Z}[1 / l]), M\left(\mathbf{Q}_{l} / \mathbf{Z}_{l}\right)$ are Moore spectra, we get a commutative diagram of homotopy fibre sequences

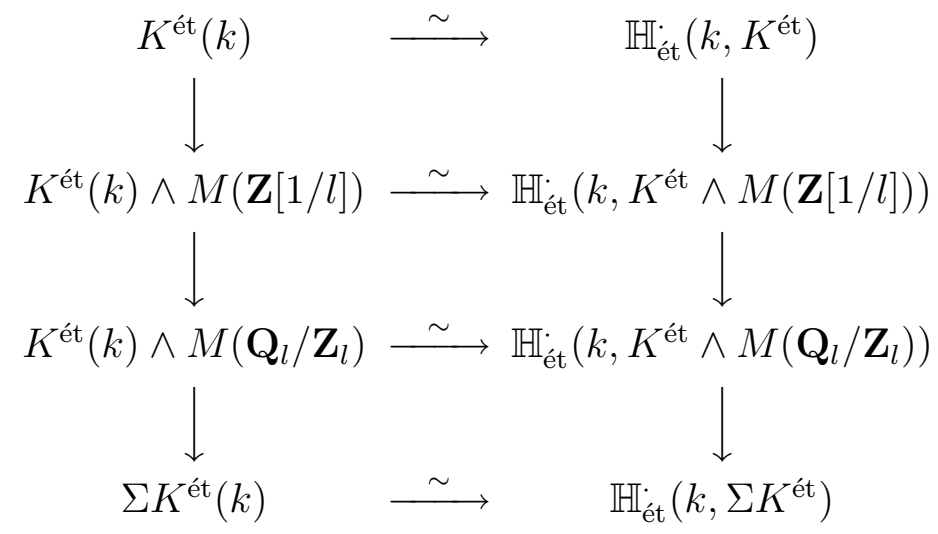

and the bottom square shows that the diagram of edge homomorphisms

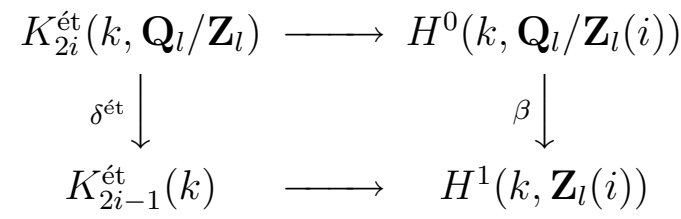

commutes, where $\delta^{\text {ét }}$ is the Bockstein coming from (4.1). ${ }^{2}$

In view of the commutative diagram

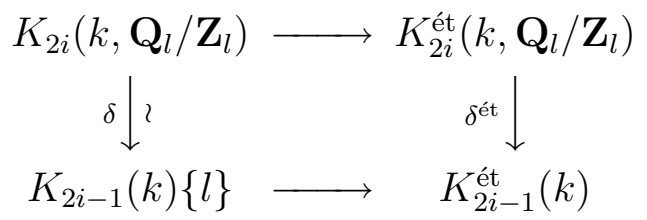

we are reduced to showing that the composition of $\delta^{-1}$ with the map $K_{2 i}\left(k, \mathbf{Q}_{l} / \mathbf{Z}_{l}\right) \rightarrow K_{2 i}^{\text {ét }}\left(k, \mathbf{Q}_{l} / \mathbf{Z}_{l}\right) \rightarrow H^{0}\left(k, \mathbf{Q}_{l} / \mathbf{Z}_{l}(i)\right)$ coincides with the map defined at the beginning of Section 2. For this, we may further replace $k$ by its algebraic closure $\bar{k}$ : then it is clear by construction (see [5, Proofs of Prop. 5.1 and Prop. 5.2] for the identification of the $E_{2}$-terms in the Dwyer-Friedlander spectral sequences).

For $i>0$ and if $X$ is essentially of finite type over $\mathbf{Z}[1 / l]$ or a $p$-adic field, we have $H_{\text {ét }}^{0}\left(X, \mathbf{Z}_{l}(i)\right)=0$ and this yields canonical homomorphisms

$$
c h_{i+1}: K_{2 i}(X) \rightarrow K_{2 i}^{\text {ét }}(X) \rightarrow H_{\text {ét }}^{2}\left(X, \mathbf{Z}_{l}(i+1)\right)
$$

which are natural in $X$.

\footnotetext{
${ }^{2}$ Note that, for any Noetherian site $X$ of finite cohomological dimension, any sheaf of spectra $F$ on $X$ and any spectrum $T$, the natural map $\mathbb{H}^{\cdot}(X, F) \wedge T \rightarrow$ $\mathbb{H}^{\cdot}(X, F \wedge T)$ is a weak equivalence.
} 
For $X=\operatorname{Spec} F$ with $F$ a finite extension of $\mathbf{Q}_{p}$, we get in this way a composite map

$$
K_{2 i}(F) \rightarrow H^{2}\left(F, \mathbf{Z}_{l}(i+1)\right) \rightarrow H^{2}(F, \mathbf{Z} / m(i+1))
$$

when $m \mid m_{i}(F)$.

Composing with the inverse of the isomorphism $\theta_{m}$ of $(3.2)$, we get a homomorphism

$$
h_{m}: K_{2 i}(F) \rightarrow H_{\text {ét }}^{0}(F, \mathbf{Z} / m(i)) .
$$

By Tate duality and Lemma 3.1, these homomorphisms are related as follows:

4.2. Lemma. Let $m\left|m^{\prime}\right| m_{i}(F)$. Then $h_{m}=\frac{m^{\prime}}{m} h_{m^{\prime}}$.

We shall write $h^{(i)}$ for the composition of $h_{m_{i}(F)}$ with the isomorphism $H^{0}\left(F, \mathbf{Z} / m_{i}(F)(i)\right) \stackrel{\sim}{\rightarrow} H^{0}\left(F, \mathbf{Q}_{l} / \mathbf{Z}_{l}(i)\right)$.

Suppose $X=\operatorname{Spec} \mathbf{R}$. If $l$ is odd, we define $h^{(i)}$ to be the zero map. If $l=2$, we cannot define a map $h^{(i)}$ as above by the same method. However, by Suslin [25, Th. 4.9], $K_{2 i}(\mathbf{R})$ is uniquely divisible for $i \not \equiv 1$ $(\bmod 4)(i>0)$ and is the direct sum of a group of order 2 and a uniquely divisible group for $i \equiv 1(\bmod 4)$. We define $h^{(i)}$ to be 0 for $i \not \equiv 1(\bmod 4)$ and to be the unique nontrivial homomorphism to $\mathbf{Z} / 2=H^{0}\left(\mathbf{R}, \mathbf{Q}_{2} / \mathbf{Z}_{2}(i)\right)$ for $i \equiv 1(\bmod 4)$.

Coming back to our number field $K$, we get for each noncomplex place $v$ a homomorphism

$$
h^{(i)}(v): K_{2 i}(K) \rightarrow H^{0}\left(K_{v}, \mathbf{Q}_{l} / \mathbf{Z}_{l}(i)\right) .
$$

4.3. Theorem (Moore [17, Th. 7.4] for $i=1$, Banaszak [2, Th. 1] for $l$ odd). Let $m_{i}=m_{i}(K)$ and, for any noncomplex place $v$ of $K$, $m_{i}(v)=m_{i}\left(K_{v}\right)$. Then, at least in the following cases:

- $l$ is odd

- $l=2$ and $K$ is totally imaginary

the sequence

$$
\begin{aligned}
K_{2 i}(K)\{l\} \stackrel{\left(h^{(i)}(v)\right)}{\longrightarrow} \underset{\text { v noncomplex }}{\bigoplus} H^{0}\left(K_{v}, \mathbf{Q}_{l} / \mathbf{Z}_{l}(i)\right) & \\
& \stackrel{\left(\frac{m_{i}(v)}{m_{i}}\right)}{\longrightarrow} \quad H^{0}\left(K, \mathbf{Q}_{l} / \mathbf{Z}_{l}(i)\right) \rightarrow 0
\end{aligned}
$$

is exact, where $\frac{m_{i}(v)}{m_{i}}$ is interpreted as 0 if $m_{i}(v)$ is infinite (this happens exactly when $v$ is real and $i$ is even). 
(The exactness will not be used in the proof of Theorems 2.6 and 2.8: only the fact that this sequence is a complex.)

For $l$ odd, this theorem is due to Banaszak (op. cit.), but he does not make explicit the constants $\frac{m_{i}(v)}{m_{i}}$ which are crucial for the sequel. We give an alternate, slightly simpler prof.

Proof. We shall get this exact sequence as a direct limit of exact sequences

$$
K_{2 i}\left(O_{S}\right)\{l\} \stackrel{\left(h^{(i)}(v)\right)}{\longrightarrow} \bigoplus_{v \in S} H^{0}\left(K_{v}, \mathbf{Q}_{l} / \mathbf{Z}_{l}(i)\right) \stackrel{\left(\frac{m_{i}(v)}{m_{i}}\right)}{\longrightarrow} H^{0}\left(K, \mathbf{Q}_{l} / \mathbf{Z}_{l}(i)\right) \rightarrow 0
$$

where $S$ runs through the finite sets of primes of $K$ containing all infinite primes and all primes dividing $l$.

For such an $S$, as long as $c d_{l}\left(O_{S}\right)<\infty$ we have the maps (4.2) at our disposal: this happens exactly when either $l$ is odd or $l=2$ and $K$ is totally imaginary. Hence a commutative diagram in these cases

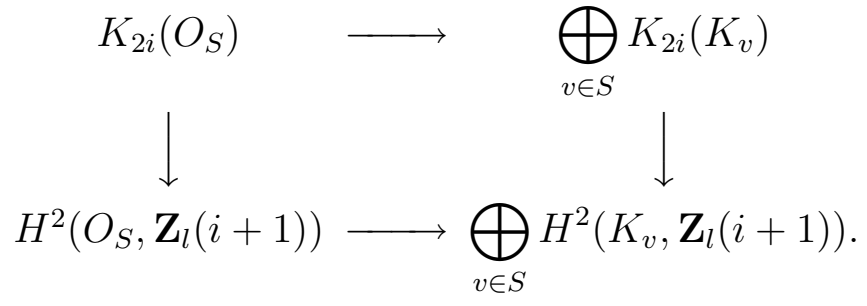

In this diagram the left vertical map is surjective by Dwyer-Friedlander [5, Th. 8.7] (assuming $\sqrt{-1} \in K$ if $l=2$ ) and by Rognes-Weibel [22] for $l=2$ in general. Hence it suffices to prove that the sequence

$$
\begin{aligned}
H^{2}\left(O_{S}, \mathbf{Z}_{l}(i+1)\right) & \rightarrow \bigoplus_{v \in S} H^{2}\left(K_{v}, \mathbf{Z}_{l}(i+1)\right) \\
& \simeq \bigoplus_{v \in S} H^{0}\left(K_{v}, \mathbf{Q}_{l} / \mathbf{Z}_{l}(i)\right) \rightarrow H^{0}\left(K, \mathbf{Q}_{l} / \mathbf{Z}_{l}(i)\right) \rightarrow 0
\end{aligned}
$$

is exact to get that the $K$-theory sequence above is exact. This follows by a passage to the inverse limit from a part of the Poitou-Tate exact sequences [16, p. 70, Th. 4.10]

$$
\begin{aligned}
H^{2}\left(O_{S}, \mathbf{Z} / l^{\nu}(i+1)\right) \rightarrow \bigoplus_{v \in S} H^{2}\left(K_{v}, \mathbf{Z} / l^{\nu}(i+1)\right) & \\
& \rightarrow H^{0}\left(K, \mathbf{Z} / l^{\nu}(-i)\right)^{*} \rightarrow 0
\end{aligned}
$$

since all groups involved in this sequences are finite, provided we identify the last map with the collection of $\frac{m_{i}(v)}{m_{i}}$. But this map is the dual 
of the natural map

$$
H^{0}\left(K, \mathbf{Z} / l^{\nu}(-i)\right) \rightarrow \prod_{v \in S} H^{0}\left(K_{v}, \mathbf{Z} / l^{\nu}(-i)\right)
$$

hence the claim by Lemma 3.1.

4.4. Remark. The only use we make of the surjectivity results of Dwyer-Friedlander and Rognes-Weibel is to prove that the complex of Theorem 4.3 is exact. For $l=2$ and $K$ formally real, it is likely that the sequence of Theorem 4.3 is still a complex; however we cannot use the maps (4.2) to prove it in this case. On the other hand it is unlikely that this sequence is still exact. The correct point of view would be to replace algebraic $K$-groups by motivic cohomology groups (defined for example by means of Bloch's cycle complexes, generalised to the case of Dedekind domains by Levine). Then the analogue of (4.2) is easy to construct in full generality. The fact that the resulting complex is exact is a consequence of the Milnor conjecture (Voevodsky). The defect of exactness for algebraic $K$-theory would correspond to the difference between the latter and motivic cohomology in the real case (see [12]).

To make this approach compatible with the current one, however, would involve comparing the natural map from motivic cohomology to étale cohomology with the edge homomorphism (4.2) of DwyerFriedlander. This appears quite nontrivial and would go far beyond the relatively elementary approach of this paper.

\section{Proof of Theorem 2.6}

5.1. Definition. Let $F$ be a local field, $a \in K_{2 i-1}(F)$ and $f \in F^{*}$. We set

$$
(a, f)=h^{(i)}(a \cdot\{f\}) \in H^{0}\left(F, \mathbf{Q}_{l} / \mathbf{Z}_{l}(i)\right) .
$$

This is the generalised Hilbert symbol.

If $\mathfrak{p}$ is a prime of $K$ and $a \in K_{2 i-1}(K), f \in K^{*}$, we set

$$
(a, f)_{\mathfrak{p}}=\left(\phi_{*} a, \phi_{*} f\right)
$$

where $\phi$ is the inclusion $K \rightarrow K_{\mathfrak{p}}$.

5.2. Proposition. Let $p$ be a prime number $\neq l$ and $F$ be a finite extension of $\mathbf{Q}_{p}$, with normalised valuation $v$, valuation ring $O_{F}$ and residue field $k$. Let $m$ be the order of $H^{0}\left(k, \mathbf{Q}_{l} / \mathbf{Z}_{l}(i)\right)$ and $q$ the cardinality of 
$k$. Then, for any $f \in F^{*}$, the diagram

$$
\begin{array}{ccc}
K_{2 i-1}\left(O_{F}\right) & \stackrel{\cdot\{f\}}{\longrightarrow} & K_{2 i}\left(O_{F}\right) \\
v(f) i_{F}^{*} \downarrow & h^{(i)} \downarrow \\
H^{0}\left(k, \mathbf{Q}_{l} / \mathbf{Z}_{l}(i)\right) & \stackrel{\frac{m}{q^{i}-1} s}{\longleftarrow} & H^{0}\left(F, \mathbf{Q}_{l} / \mathbf{Z}_{l}(i)\right)
\end{array}
$$

commutes, where $s$ is the map of (3.3) and $i_{F}^{*}$ is the map of (2.5).

Proof. This follows from the functoriality and multiplicative properties of the Dwyer-Friedlander spectral sequence, and Proposition 3.4.

5.3. Corollary. Let $K$ be a number field and $\mathfrak{p}$ a prime of $K$ not dividing $l$. Let $i>0$. Then, for any $(a, f) \in K_{2 i-1}\left(R_{K}\right) \times K^{*}$, one has

$$
\frac{m_{i}(\mathfrak{p})}{m_{i}}(a, f)_{\mathfrak{p}}=v_{\mathfrak{p}}(f)\left(\frac{a}{\mathfrak{p}}\right) .
$$

5.4. Lemma. Let $F$ be a finite extension of $\mathbf{Q}_{l}$. Then, for any $a \in$ $K_{2 i-1}\left(O_{F}\right)$, the map

$$
\begin{aligned}
F^{*} & \rightarrow H^{0}\left(F, \mathbf{Q}_{l} / \mathbf{Z}_{l}(i)\right) \\
f & \mapsto(a, f)
\end{aligned}
$$

is continuous.

Proof. This is obvious, since the target group is a finite l-group. (Use the fact that the topology of $U_{F}^{(1)}$ coincides with the topology given by $l^{n}$-th powers.)

5.5. Theorem (Reciprocity). Suppose that either $l$ is odd or $K$ is totally imaginary. Then, for any $a \in K_{2 i-1}\left(R_{K}\right)$ and any $f \in K^{*}$ with divisor prime to $l$, we have

$$
\left(\frac{a}{(f)}\right)=-\sum_{\mathfrak{l} l} \frac{m_{i}(\mathfrak{l})}{m_{i}}(a, f)_{\mathfrak{l}}
$$

(This is a higher analogue of formula (**) of [4, ex. 2.10 p. 353].)

Proof. Apply Theorem 4.3 to $a \cdot\{f\}$ and use Corollary 5.3.

Proof of Theorem 2.6. This follows immediately from Theorem 5.5 and Lemma 5.4.

5.6. Remark. The proof of Theorem 2.6 gives an easy way to estimate the moduli $\mathfrak{m}_{i}$ : for $\mathfrak{l} \mid l$, it suffices to choose $v_{\mathfrak{l}}\left(\mathfrak{m}_{i}\right)$ large enough so that

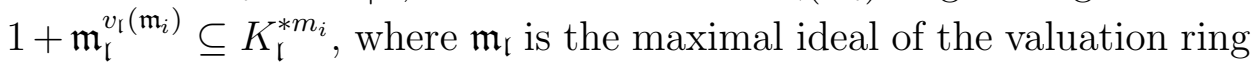
of $K_{\mathfrak{l}}$. This shows that $\mathfrak{m}_{i}$ depends on $i$, at least a priori. 


\section{SOMME HORRIBLE COMPUTATIONS}

6.1. Lemma. a) Let $u \in U^{(1)}$, where $U^{(1)}$ is the group of principal units of $\mathbf{C}_{l}$. Suppose that $v(u-1) \geq \inf \left(\frac{1}{l-2}, \frac{3}{2}\right)$, where $v$ is the valuation of $\mathbf{C}_{l}$. Then we have

$$
u \equiv \exp (u-1) \quad\left(\bmod \frac{(u-1)^{2}}{2} \mathbf{O}_{l}\right)
$$

where $\mathbf{O}_{l}$ is the valuation ring of $\mathbf{C}_{l}$.

b) Let $u$ be a unit of $\mathbf{C}_{l}$. Assume that $v\left(u^{d}-1\right) \geq \inf \left(\frac{1}{l-2}, \frac{3}{2}\right)$ for a suitable integer d prime to $l$. Then, for any integer $n>0$ multiple of $d$, we have

$$
u \equiv \exp \left(\frac{u^{n}-1}{n}\right)\left(\bmod ^{*}\left(U^{\left(v_{1}\right)}\right)^{l^{a}}\right)
$$

where $v_{1}=v\left(\frac{\left(u^{d}-1\right)^{2}}{2}\right)$ and $l^{a}$ is the largest power of $l$ dividing $n$. c) Suppose $l=2$. Let $u$ be a unit of $\mathbf{Z}_{2}$ and let $\nu=v_{2}\left(u^{2}-1\right)-1$. Then, for any integer $n>0$, we have

$$
u \equiv(-1)^{\frac{u-1}{2}} \exp \left(\frac{u^{2 n}-1}{2 n}\right)\left(\bmod ^{*} U^{2^{a+2 \nu-1}}\right)
$$

where $2^{a}$ is the largest power of 2 dividing $n$.

(We shall use this lemma only for $u \in \mathbf{Q}_{l}$.)

Proof. a) Write

$$
\exp (u-1)=u+\frac{(u-1)^{2}}{2}+\cdots+\frac{(u-1)^{k}}{k !}+\ldots
$$

First note that, if $v(u-1) \geq \inf \left(\frac{1}{l-2}, \frac{3}{2}\right)$, then $v(u-1)>\frac{1}{l-1}$ and the exponential series converges. It is now sufficient to show that, under the former assumption, $v\left(\frac{(u-1)^{k}}{k !}\right) \geq v\left(\frac{(u-1)^{2}}{2}\right)$ for all $k>2$. The left hand side is

$$
v\left(\frac{(u-1)^{k}}{k !}\right)=k v(u-1)-\frac{k-S_{l}(k)}{l-1}
$$

where $S_{l}(k)$ is the sum of the digits of $k$ written in base $l$. We now have

$v\left(\frac{(u-1)^{k}}{k !}\right)-v\left(\frac{(u-1)^{2}}{2}\right)=(k-2)\left(v(u-1)-\frac{1}{l-1}\right)+\frac{S_{l}(k)-2}{l-1}$. 
If $k$ is not a power of $l, S_{l}(k) \geq 2$ and this is $\geq 0$ by assumption. If $k$ is a power of $l$ with $l$ odd, the right hand side equals

$$
\begin{array}{r}
(k-2)\left(v(u-1)-\frac{1}{l-1}\right)-\frac{1}{l-1} \geq(l-2)\left(v(u-1)-\frac{1}{l-1}\right)-\frac{1}{l-1} \\
=(l-2) v(u-1)-1
\end{array}
$$

and this is still $\geq 0$. Finally, if $l=2$ and $k$ is a power of 2 , then

$$
(k-2)(v(u-1)-1)-1 \geq 2(v(u-1)-1)-1
$$

and this is $\geq 0$ again.

b) We may write $u=\zeta u_{0}$, with $\zeta \in \mu_{d}$ and $u_{0} \in U^{(1)}$. Since $\zeta \equiv 1\left(\bmod ^{*} U^{l^{a}}\right)$, we are reduced to the case $u \in U^{(1)}$.

Applying a) to $u^{n}$ instead of $u$, we get

$$
u^{n} \equiv \exp \left(u^{n}-1\right) \quad\left(\bmod \frac{\left(u^{n}-1\right)^{2}}{2} \mathbf{O}_{l}\right)
$$

or equivalently

$$
u^{n} \equiv \exp \left(u^{n}-1\right)\left(\bmod ^{*} U^{(v)}\right)
$$

where

$$
v=v\left(\frac{\left(u^{n}-1\right)^{2}}{2}\right)=2 v(n)+v_{1}
$$

by Lemma 1.5 , with $v_{1}=v\left(\frac{(u-1)^{2}}{2}\right)$.

Recall the following well-known fact: if $s>\frac{1}{l-1}$, raising to the $l$-th power induces an isomorphism $U^{(s)} \stackrel{\sim}{\rightarrow} U^{(s+1)}$. Writing $n=l^{a} n_{0}$ with $n_{0}$ prime to $l$, we find first

$$
u^{n_{0}} \equiv \exp \left(\frac{u^{n}-1}{l^{a}}\right)\left(\bmod ^{*} U^{\left(v_{1}+a\right)}\right)
$$

and, since $U^{(1)}$ is uniquely $n_{0}$-divisible

$$
u \equiv \exp \left(\frac{u^{n}-1}{n}\right)\left(\bmod ^{*} U^{\left(v_{1}+a\right)}\right)
$$

which may be rewritten

$$
u \equiv \exp \left(\frac{u^{n}-1}{n}\right)\left(\bmod ^{*}\left(U^{\left(v_{1}\right)}\right)^{n}\right) .
$$

c) Note that $u^{2} \equiv 1(\bmod 8)$. Applying b) to $u^{2}$, we get

$$
u^{2} \equiv \exp \left(\frac{u^{2 n}-1}{n}\right)\left(\bmod ^{*}\left(U_{\mathbf{Z}_{2}}^{(2 \nu+1)}\right)^{n}\right)
$$

where $U_{\mathbf{Z}_{2}}^{(r)}:=U^{(r)} \cap \mathbf{Z}_{2}$. Since $u \equiv(-1)^{\frac{u-1}{2}}\left(\bmod U_{\mathbf{Z}_{2}}^{(3)}\right)$ and $U_{\mathbf{Z}_{2}}^{(2 \nu+1)}=$ $\left(U_{\mathbf{Z}_{2}}\right)^{2^{2 \nu-1}}$, we get the desired congruence. 
6.2. Lemma. Let $E / F$ be a finite extension of finite extensions of $\mathbf{Q}_{p}$ and $i>0$. Then

a) The diagram

$$
\begin{aligned}
& K_{2 i}(E) \stackrel{h^{(i)}(E)}{\longrightarrow} H^{0}\left(E, \mathbf{Q}_{l} / \mathbf{Z}_{l}(i)\right) \\
& N_{L / K} \downarrow \quad \frac{m_{i}(E)}{m_{i}(E)} \downarrow \\
& K_{2 i}(F) \stackrel{h^{(i)}(F)}{\longrightarrow} H^{0}\left(F, \mathbf{Q}_{l} / \mathbf{Z}_{l}(i)\right)
\end{aligned}
$$

commutes.

b) If $p \neq l$, the diagram

$$
\begin{array}{ll}
K_{2 i-1}\left(O_{E}\right) \stackrel{i_{E}^{*}}{\longrightarrow} H^{0}\left(\ell, \mathbf{Q}_{l} / \mathbf{Z}_{l}(i)\right) \\
N_{L / K} \downarrow \\
K_{2 i-1}\left(O_{F}\right) \stackrel{|\ell| i^{i}-1}{\mid \frac{i_{F}^{*}-1}{}} \downarrow \\
H^{0}\left(k, \mathbf{Q}_{l} / \mathbf{Z}_{l}(i)\right)
\end{array}
$$

commutes, where $k$ (resp. $\ell$ ) is the residue field of $F$ (resp. of $E$ ) and $e$ is the ramification index of $E / F$.

Proof. a) By the covariance of the Dwyer-Friedlander spectral sequence for proper morphisms, the diagram

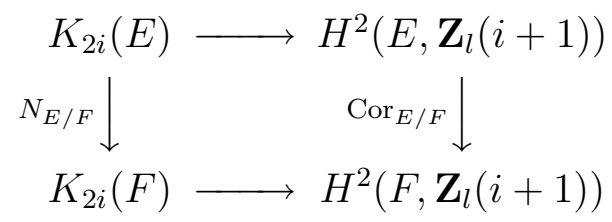

commutes. On the other hand, Tate duality exchanges corestriction and restriction. The claim then follows from Lemma 3.1.

b) This reduces to proving the commutation of the two diagrams

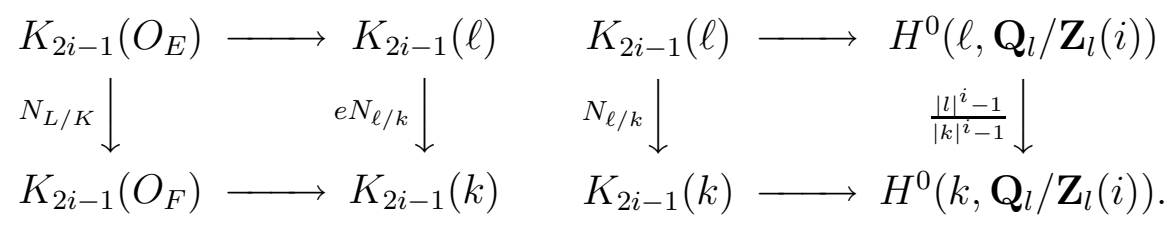

The first one is obvious in view of the functoriality of algebraic $K$ theory, and the second one follows from Lemma 1.4.

6.3. Lemma. a) With notation as in Lemma 6.2, let $a \in K_{2 i-1}(E)$ and $f \in F^{*}$. Then

$$
\left(N_{E / F}(a), f\right)=\frac{m_{i}(E)}{m_{i}(F)}(a, f) .
$$


b) Let $L / K$ be an extension of number fields, $a \in K_{2 i-1}\left(R_{L}\right)$ and $\mathfrak{a} a$ fractional ideal of $R_{K}$. Then

$$
\left(\frac{N_{L / K}(a)}{\mathfrak{a}}\right)=\frac{m_{i}(L)}{m_{i}(K)}\left(\frac{a}{\mathfrak{a} R_{L}}\right) .
$$

Proof. a) immediately follows from Lemma 6.2 a) and the projection formula in algebraic $K$-theory. We deduce b) from Lemma $6.2 \mathrm{~b}$ ) as follows: first we reduce to the case where $\mathfrak{a}=\mathfrak{p}$ is prime. Now, developing both sides, we have to prove the identity

$$
\frac{N(\mathfrak{p})^{i}-1}{m_{i}(K)} i_{\mathfrak{p}}^{*} N_{L / K}(a)=\frac{m_{i}(L)}{m_{i}(K)} \sum_{\mathfrak{P} \mid \mathfrak{p}} e_{\mathfrak{P}} \frac{N(\mathfrak{P})^{i}-1}{m_{i}(L)} i_{\mathfrak{P}}^{*} a .
$$

But Lemma 6.2 a) give the identity

$$
i_{\mathfrak{p}}^{*} N_{L / K}(a)=\sum_{\mathfrak{P} \mid \mathfrak{p}} e_{\mathfrak{P}} \frac{N(\mathfrak{P})^{i}-1}{N(\mathfrak{p})^{i}-1} i_{\mathfrak{P}}^{*} a
$$

which immediately implies the former.

6.4. Lemma. Let $L / K$ be an extension of number fields and $\mathfrak{p}$ a prime ideal of $R_{K}$. Then

a) One has

$$
\operatorname{deg}_{l}\left(\mathfrak{p} R_{L}\right)=\frac{m_{i}(K)}{m_{i}(L)} \sum_{\mathfrak{P} \mid \mathfrak{p}} e_{\mathfrak{P}}\left|f_{\mathfrak{P}}\right|_{l}^{-1} \operatorname{deg}_{l}(\mathfrak{p})
$$

where $e_{\mathfrak{P}}$ and $f_{\mathfrak{P}}$ denote respectively the ramification index and the residue degree at $\mathfrak{P}$. When $L / K$ is Galois, this formula simplifies in

$$
\operatorname{deg}_{l}\left(\mathfrak{p} R_{L}\right)=\left(f_{\mathfrak{p}}^{0}\right)^{-1}\left[L_{0}: K_{0}\right]^{0} \frac{[L: K]}{\left[L_{0}: K_{0}\right]} \operatorname{deg}_{l}(\mathfrak{p})
$$

where $f_{\mathfrak{p}}:=f_{\mathfrak{P}}$ for any prime $\mathfrak{P} \mid \mathfrak{p}$ and, for an integer $a \neq 0$, $a^{0}$ denotes the prime-to-l part of a and $K_{0}, L_{0}$ denote respectively the maximal l-cyclotomic subfield of $K$ and $L$. Moreover

$$
\frac{m_{i}(\mathfrak{P})}{f_{\mathfrak{p}} i}= \begin{cases}\left(i^{0}\right)^{-1} m_{l-1}(L)[L: K]^{-1} \operatorname{deg}_{l}\left(\mathfrak{p} R_{L}\right) & \text { if } l>2 \\ \left(i^{0}\right)^{-1} m_{2}(L)[L: K]^{-1} \operatorname{deg}_{l}\left(\mathfrak{p} R_{L}\right) / 2 & \text { if } l=2 .\end{cases}
$$

b) Let $a \in K_{2 i-1}\left(R_{L}\right)$. If $L / K$ is Galois, one has

$$
2\left(\left(\frac{N_{L / K}(a)}{\mathfrak{p}}\right)^{(l)}-f_{\mathfrak{p}}^{0} \frac{m_{i}(L)}{m_{i}(K)}\left(\frac{a}{\mathfrak{p} R_{L}}\right)^{(l)}\right)=0 .
$$


Proof. a) We have

$$
\begin{aligned}
& \operatorname{deg}_{l}\left(\mathfrak{p} R_{L}\right)=\sum_{\mathfrak{P} \mid \mathfrak{p}} e_{\mathfrak{P}} \operatorname{deg}_{l}(\mathfrak{P})=\sum_{\mathfrak{P} \mid \mathfrak{p}} e_{\mathfrak{P}} \frac{m_{i}(\mathfrak{P})}{m_{i}(L)} \\
& =\frac{m_{i}(K)}{m_{i}(L)} \sum_{\mathfrak{P} \mid \mathfrak{p}} e_{\mathfrak{P}} \frac{m_{i}(\mathfrak{P})}{m_{i}(\mathfrak{p})} \frac{m_{i}(\mathfrak{p})}{m_{i}(K)}=\frac{m_{i}(K)}{m_{i}(L)} \sum_{\mathfrak{P} \mid \mathfrak{p}} e_{\mathfrak{P}}\left|f_{\mathfrak{P}}\right|_{l}^{-1} \operatorname{deg}_{l}(\mathfrak{p}) .
\end{aligned}
$$

Suppose now that $L / K$ is Galois. Then all $f_{\mathfrak{P}}$ are equal (to $f_{\mathfrak{p}}$, by definition), and we may write

$$
\begin{gathered}
\frac{m_{i}(K)}{m_{i}(L)} \sum_{\mathfrak{P} \mid \mathfrak{p}} e_{\mathfrak{P}}\left|f_{\mathfrak{P}}\right|_{l}^{-1} \operatorname{deg}_{l}(\mathfrak{p})=\frac{m_{i}(K)}{m_{i}(L)}\left(f_{\mathfrak{p}}^{0}\right)^{-1} \sum_{\mathfrak{P} \mid \mathfrak{p}} e_{\mathfrak{P}} f_{\mathfrak{P}} \operatorname{deg}_{l}(\mathfrak{p}) \\
=\frac{m_{i}(K)}{m_{i}(L)}\left(f_{\mathfrak{p}}^{0}\right)^{-1}[L: K] \operatorname{deg}_{l}(\mathfrak{p}) \\
=\frac{m_{i}\left(K_{0}\right)}{m_{i}\left(L_{0}\right)}\left[L_{0}: K_{0}\right]\left(f_{\mathfrak{p}}^{0}\right)^{-1} \frac{[L: K]}{\left[L_{0}: K_{0}\right]} \operatorname{deg}_{l}(\mathfrak{p}) \\
=\left[L_{0}: K_{0}\right]^{0}\left(f_{\mathfrak{p}}^{0}\right)^{-1} \frac{[L: K]}{\left[L_{0}: K_{0}\right]} \operatorname{deg}_{l}(\mathfrak{p})
\end{gathered}
$$

(the equality $\frac{m_{i}\left(K_{0}\right)}{m_{i}\left(L_{0}\right)}\left[L_{0}: K_{0}\right]=\left[L_{0}: K_{0}\right]^{0}$ is easy to check).

For the second formula,

$$
\begin{array}{r}
\frac{m_{i}(\mathfrak{P})}{f_{\mathfrak{p}} i}=\frac{m_{i}(L)}{f_{\mathfrak{p}} i} \\
\operatorname{deg}_{l}(\mathfrak{P})=\frac{m_{i}(L)}{e_{\mathfrak{P}} f_{\mathfrak{p}} i} \operatorname{deg}_{l}\left(\mathfrak{p} R_{L}\right)=\frac{m_{i}(L)}{[L: K] i} \operatorname{deg}_{l}\left(\mathfrak{p} R_{L}\right) \\
= \begin{cases}\left(i^{0}\right)^{-1} m_{l-1}(L)[L: K]^{-1} \operatorname{deg}_{l}\left(\mathfrak{p} R_{L}\right) & \text { if } l>2 \\
\left(i^{0}\right)^{-1} m_{2}(L)[L: K]^{-1} \operatorname{deg}_{l}\left(\mathfrak{p} R_{L}\right) / 2 & \text { if } l=2 .\end{cases}
\end{array}
$$

b) From Lemma 6.3 b) we have

$$
\begin{gathered}
\left(\frac{N_{L / K}(a)}{\mathfrak{p}}\right)^{(l)}=\frac{m_{i}(\mathfrak{p})}{N(\mathfrak{p})^{i}-1}\left(\frac{N_{L / K}(a)}{\mathfrak{p}}\right)=\frac{m_{i}(\mathfrak{p})}{N(\mathfrak{p})^{i}-1} \frac{m_{i}(L)}{m_{i}(K)}\left(\frac{a}{\mathfrak{p} R_{L}}\right) \\
=\frac{m_{i}(\mathfrak{p})}{N(\mathfrak{p})^{i}-1} \frac{m_{i}(L)}{m_{i}(K)} \sum_{\mathfrak{P} \mid \mathfrak{p}} e_{\mathfrak{P}}\left(\frac{a}{\mathfrak{P}}\right) \\
=\frac{m_{i}(\mathfrak{p})}{N(\mathfrak{p})^{i}-1} \frac{m_{i}(L)}{m_{i}(K)} \sum_{\mathfrak{P} \mid \mathfrak{p}} e_{\mathfrak{P}} \frac{N(\mathfrak{P})^{i}-1}{m_{i}(\mathfrak{P})}\left(\frac{a}{\mathfrak{P}}\right)^{(l)} .
\end{gathered}
$$


Since $L / K$ is Galois, we have $N(\mathfrak{P})=N(\mathfrak{p})^{f_{\mathfrak{P}}}$ for any $\mathfrak{P} \mid \mathfrak{p}$ and, by Lemma 1.5

$$
\frac{N(\mathfrak{P})^{i}-1}{N(\mathfrak{p})^{i}-1} \equiv f_{\mathfrak{P}} \quad\left(\bmod \frac{N(\mathfrak{P})^{i}-1}{2} \mathbf{Z}_{l}\right) .
$$

Moreover, we have

$$
f_{\mathfrak{P}}=f_{\mathfrak{p}}=f_{\mathfrak{p}}^{0} \frac{m_{i}(\mathfrak{P})}{m_{i}(\mathfrak{p})}
$$

for any $\mathfrak{P} \mid \mathfrak{p}$. Hence

$$
\begin{aligned}
2\left(\frac{N_{L / K}(a)}{\mathfrak{p}}\right)^{(l)} & =2 f_{\mathfrak{p}} \frac{m_{i}(\mathfrak{p})}{m_{i}(\mathfrak{P})} \frac{m_{i}(L)}{m_{i}(K)} \sum_{\mathfrak{P} \mid \mathfrak{p}} e_{\mathfrak{P}}\left(\frac{a}{\mathfrak{P}}\right)^{(l)} \\
= & 2 f_{\mathfrak{p}}^{0} \frac{m_{i}(L)}{m_{i}(K)} \sum_{\mathfrak{P} \mid \mathfrak{p}} e_{\mathfrak{P}}\left(\frac{a}{\mathfrak{P}}\right)^{(l)}=2 f_{\mathfrak{p}}^{0} \frac{m_{i}(L)}{m_{i}(K)}\left(\frac{a}{\mathfrak{p} R_{K}}\right)^{(l)}
\end{aligned}
$$

as requested.

\section{Proof of Theorem 2.8}

By additivity of both sides, we may assume that $r$ is a prime $p \neq l$. Note first that by Lemma 6.4 we have

$$
\frac{\operatorname{deg}_{l}\left(p R_{K}\right)}{2\left[K: K_{0}\right]}=\left(f_{p}^{0}\right)^{-1} \frac{1}{2}\left[K_{0}: \mathbf{Q}\right] \operatorname{deg}_{l}(p \mathbf{Z})
$$

where $f_{p}$ is the residue degree at $p$ in the Galois extension $K / \mathbf{Q}$. This is an $l$-integer for $l>2$. This is still the case for $l=2$ except perhaps if $K_{0}=\mathrm{Q}$. But $K_{0}=\mathrm{Q}$ is real, hence excluded from the statement of Theorem 2.8.

Suppose first that $l$ is odd. We are going to prove Theorem 2.8 a) with

$$
s(a)=-\left(i^{0}\right)^{-1}\left(N_{K / K_{0}}(a), \exp \left(\frac{l}{\delta}\right)\right)_{\mathfrak{l}}
$$

where $\left[K_{0}: \mathbf{Q}\right]=\delta l^{n-1}, \mathfrak{l}$ is the unique prime of $K_{0}$ dividing $l$ and $i^{0}$ is the largest divisor of $i$ that is prime to $l$. By Lemma 6.4, we immediately reduce to the case where $K=K_{0}$ (notice that $m_{i}(K)=$ $m_{i}\left(K_{0}\right)$ for all $\left.i\right)$.

Then $l$ is the only ramified prime in $K / \mathbf{Q}$ and it is totally ramified, so that

$$
m_{i}=m_{i}(\mathfrak{l})=l^{n+v_{l}(i)} \text { if } \delta \mid i
$$

where $\mathfrak{l}$ is the unique prime of $K$ above $l$. 
By Lemma 6.1 b), we have

$$
p \equiv \exp \left(\frac{p^{f i}-1}{f i}\right) \quad\left(\bmod \left(U^{\left(v_{1}\right)}\right)^{|f i|_{l}^{-1}}\right)
$$

where $v_{1}=2 v_{l}\left(p^{l-1}-1\right)=2 v_{l}\left(m_{l-1}(p \mathbf{Z})\right)$. Since $U^{\left(v_{1}\right)}=\left(U^{(1)}\right)^{l^{v_{1}-1}}$, this gives

$$
p \equiv \exp \left(\frac{p^{f i}-1}{f i}\right) \quad\left(\bmod \left(U^{\left|f i m_{l-1}(p \mathbf{Z})^{2} / l\right|_{l}^{-1}}\right) .\right.
$$

On the other hand,

$$
\begin{aligned}
& v_{l}\left(f i m_{l-1}(p \mathbf{Z})^{2} / l\right)-v_{l}\left(m_{i}\right)=v_{l}\left(f m_{l-1}(p \mathbf{Z})^{2} / l\right)-v_{l}\left(m_{l-1}\right) \\
& =v_{l}\left(\frac{m_{l-1}(\mathfrak{p})}{m_{l-1}(p \mathbf{Z})} \frac{m_{l-1}(p \mathbf{Z})^{2}}{l}\right)-v_{l}\left(m_{l-1}\right)=v_{l}\left(\frac{m_{l-1}(\mathfrak{p})}{m_{l-1}} \frac{m_{l-1}(p \mathbf{Z})}{l}\right) \\
& \left.=v_{l}\left(\operatorname{deg}_{l}(\mathfrak{p})\right)+v_{l}\left(\frac{m_{l-1}(p \mathbf{Z})}{l}\right)\right) \geq 0 .
\end{aligned}
$$

Hence, using Theorem 5.5, we get

$$
\begin{aligned}
\left(\frac{a}{p R_{K}}\right)^{(l)} & =\frac{m_{i}(\mathfrak{p})}{N(\mathfrak{p})^{i}-1}\left(\frac{a}{p R_{K}}\right)=-\frac{m_{i}(\mathfrak{p})}{N(\mathfrak{p})^{i}-1}(a, p)_{\mathfrak{l}} \\
= & -\frac{m_{i}(\mathfrak{p})}{p^{f i}-1}(a, p)_{\mathfrak{l}}=-\frac{m_{i}(\mathfrak{p})}{p^{f i}-1}\left(a, \exp \left(\frac{p^{f i}-1}{f i}\right)\right)_{\mathfrak{l}} \\
& =-\left(a, \exp \left(\frac{m_{i}(\mathfrak{p})}{f i}\right)\right)_{\mathfrak{l}}
\end{aligned}
$$

By Lemma 6.4 a), we have

$$
\frac{m_{i}(\mathfrak{p})}{f i}=\left(i^{0}\right)^{-1} m_{l-1}(K)[K: \mathbf{Q}]^{-1} \operatorname{deg}_{l}\left(p R_{K}\right)=\left(i^{0}\right)^{-1} \operatorname{deg}_{l}\left(p R_{K}\right) \frac{l}{\delta}
$$

hence

$$
\left(\frac{a}{p R_{K}}\right)^{(l)}=-\left(i^{0}\right)^{-1} \operatorname{deg}_{l}\left(p R_{K}\right)\left(a, \exp \left(\frac{l}{\delta}\right)\right) \mathfrak{r}
$$

as requested.

Suppose now that $l=2$. We shall prove Theorem 2.8 b) with

$$
\begin{aligned}
& s_{1}(a)=\left(N_{K / K_{0}}(a),-1\right)_{\mathfrak{l}} \\
& s_{2}(a)=-\left(i^{0}\right)^{-1}\left(N_{K / K_{0}}(a), \exp (4)\right)_{\mathfrak{r}} .
\end{aligned}
$$

As above, we reduce to $K=K_{0}$ and $r=p \neq 2$ (a prime number). Then $K$ is classified by an open subgroup $\Gamma_{K}$ of $\mathbf{Z}_{2}^{*}$. Given an integer $n \geq 1$, there are exactly three such subgroups of index $2^{n}$, namely:

- $\Gamma_{K}=\Gamma^{n}=1+2^{n+1} \mathbf{Z}_{2}$, corresponding to $K=\mathbf{Q}\left(\mu_{2^{n+1}}\right)$ (cyclotomic case); $\left(\Gamma_{K}\right)^{2}=\Gamma^{n+1}$. 
- $\Gamma_{K}=\Gamma_{+}^{n}=\mu_{2} \times\left(1+2^{n+2} \mathbf{Z}_{2}\right)$, corresponding to $K=\mathbf{Q}\left(\zeta+\zeta^{-1}\right)$ where $\zeta$ is a generator of $\mu_{2^{n+2}}(\overline{\mathbf{Q}})$ (totally real case); $\left(\Gamma_{K}\right)^{2}=$ $\Gamma^{n+2}$.

- $\Gamma_{K}=\Gamma_{-}^{n}=-1+2^{n+1} \mathbf{Z}_{2}$, corresponding to $K=\mathbf{Q}\left(\zeta-\zeta^{-1}\right)$ (non-exceptional case); $\left(\Gamma_{K}\right)^{2}=\Gamma^{n+1}$.

We include $K=\mathbf{Q}$ in the totally real series: $\Gamma_{\mathbf{Q}}=\mathbf{Z}_{2}^{*}$ and $\left(\Gamma_{\mathbf{Q}}\right)^{2}=$ $\Gamma^{2}$. Using these computations, we find that

$$
m_{2}(K)=\left(\mathbf{Z}_{2}^{*}:\left(\Gamma_{K}\right)^{2}\right)= \begin{cases}2^{n+3} & \text { if } K \text { is totally real } \\ 2^{n+2} & \text { otherwise. }\end{cases}
$$

Recall that the totally real case is excluded from the statement of Theorem 2.8. Henceforth we assume $K$ totally imaginary.

Let $p^{*}=(-1)^{\frac{p-1}{2}} p$. By Lemma $\left.6.1 \mathrm{c}\right)$, we have

$$
p \equiv(-1)^{\frac{p-1}{2}} \exp \left(\frac{\left(p^{*}\right)^{f i}-1}{f i}\right) \quad\left(\bmod U^{|f i / 2|_{2}^{-1} 2^{2 v_{1}-3}}\right)
$$

where $v_{1}=v_{2}\left(p^{2}-1\right)=v_{2}\left(m_{2}(p \mathbf{Z})\right)$. On the other hand,

$$
\begin{aligned}
v_{2}\left(f_{i m}(p \mathbf{Z})^{2} / 16\right)-v_{2}\left(m_{i}\right)= & v_{2}\left(f m_{2}(p \mathbf{Z})^{2} / 16\right)-v_{2}\left(m_{2}\right)+1 \\
=v_{2}\left(\frac{m_{2}(\mathfrak{p})}{m_{2}(p \mathbf{Z})} \frac{m_{2}(p \mathbf{Z})^{2}}{8}\right)- & v_{2}\left(m_{2}\right)=v_{2}\left(\frac{m_{2}(\mathfrak{p})}{m_{2}} \frac{m_{2}(p \mathbf{Z})}{8}\right) \\
& \left.=v_{2}\left(\operatorname{deg}_{2}(\mathfrak{p})\right)+v_{2}\left(\frac{m_{2}(p \mathbf{Z})}{8}\right)\right) \geq 0 .
\end{aligned}
$$

Suppose first that $f i$ is even or that $p \equiv 1(\bmod 4)$. Then $\left(p^{*}\right)^{f i}=$ $p^{f i}$. Using Theorem 5.5, we get

$$
\begin{aligned}
&\left(\frac{a}{p R_{K}}\right)^{(2)}=\frac{m_{i}(\mathfrak{p})}{N(\mathfrak{p})^{i}-1}\left(\frac{a}{p R_{K}}\right)=-\frac{m_{i}(\mathfrak{p})}{N(\mathfrak{p})^{i}-1}(a, p)_{\mathfrak{l}} \\
&=-\frac{m_{i}(\mathfrak{p})}{p^{f i}-1}(a, p)_{\mathfrak{l}}=-\frac{m_{i}(\mathfrak{p})}{p^{f i}-1}\left(a,(-1)^{\frac{p-1}{2}} \exp \left(\frac{p^{f i}-1}{f i}\right)\right)_{\mathfrak{l}} \\
&=-\left(a,(-1)^{\frac{p-1}{2}} \exp \left(\frac{m_{i}(\mathfrak{p})}{f i}\right)\right)_{\mathfrak{l}}
\end{aligned}
$$

By Lemma 6.4 a) and (7.1)

$$
\frac{m_{i}(\mathfrak{p})}{f i}=\left(i^{0}\right)^{-1} m_{2}(K)[K: \mathbf{Q}]^{-1} \operatorname{deg}_{2}\left(p R_{K}\right) / 2=2\left(i^{0}\right)^{-1} \operatorname{deg}_{2}\left(p R_{K}\right) \text {. }
$$

Since $\operatorname{deg}_{2}\left(p R_{K}\right)=\left(f_{p}^{0}\right)^{-1}[K: \mathbf{Q}] \operatorname{deg}_{2}(p \mathbf{Z})$, this integer is always divisible by 4 . Hence

$$
\left(\frac{a}{p R_{K}}\right)^{(2)}=\frac{p-1}{2}(a,-1)_{\mathfrak{l}}-\frac{\left.\operatorname{deg}_{2}\left(p R_{K}\right)\right)}{2}\left(i^{0}\right)^{-1}(a, \exp (4))_{\mathfrak{l}}
$$


Suppose now that $p \equiv-1(\bmod 4)$ and $f i$ is odd, hence $f=1$. The condition $f=1$ means that $p \in \Gamma_{K}$. The first condition rules out the cyclotomic case, hence (since the real case is excluded) $K$ is nonexceptional. Then the condition $i$ odd implies that $m_{i}(K)=m_{i}\left(K_{\mathfrak{l}}\right)=$ 2 , hence the symbol $(a, f)$ r has order 2 and we have

$$
\left(\frac{a}{p R_{K}}\right)^{(2)}=(a, p)_{\mathfrak{l}}=\frac{p-1}{2}(a,-1)_{\mathfrak{l}}+\left(a, p^{*}\right)_{\mathfrak{l}}
$$

Let $[K: \mathbf{Q}]=2^{n}$. We must have $p \equiv-1+2^{n+1}\left(\bmod 2^{n+2}\right)$. Then $p^{*}$ is a square in $\mathbf{Z}_{2}^{*}$ unless $n=1$, i.e. $K=\mathbf{Q}(\sqrt{-2})$ : then $p^{*} \equiv 5 \equiv \exp (4)$ $(\bmod 8)$.

On the other hand, note that $\operatorname{deg}_{2}(p \mathbf{Z})=\left|\frac{p^{2}-1}{8}\right|_{2}^{-1}$ is $>1$ exactly when $p^{*}$ is a square. This shows by inspection that $\frac{\operatorname{deg}_{2}\left(p R_{K}\right)}{2}$ is even exactly when $p^{*}$ is a square.

This discussion shows that formula (7.2) remains valid when $p \equiv-1$ $(\bmod 4)$ and $f i$ is odd.

7.1. Remarks. a) Suppose $K / \mathbf{Q} l$-cyclotomic with $[K: \mathbf{Q}]=\delta l^{n-1}$ as above. Let $\nu=v_{l}\left(p^{l-1}-1\right)$ for $l>2$ and $\nu=v_{2}\left(p^{2}-1\right)-1$ for $l=2$. By Lemma 6.4 a), we have

$$
\operatorname{deg}_{l}\left(p R_{K}\right)=\left(f_{p}^{0}\right)^{-1}[K: \mathbf{Q}]^{0} \operatorname{deg}_{l}(p \mathbf{Z})= \begin{cases}l^{\nu-1} \frac{\delta}{\operatorname{gcd}(d, \delta)} & \text { if } l>2 \\ 2^{\nu-1} & \text { if } l=2\end{cases}
$$

so that $\operatorname{deg}_{l}\left(p R_{K}\right)$ does not depend on $n$ (however it depends on $\delta$ when $l$ is odd).

b) For $l=2, \sqrt{\exp (4)} \in \mathbf{Q}_{2}(\sqrt{5})-\mathbf{Q}_{2}$ : so there is no way in general to write the summand $\frac{\left.\operatorname{deg}_{2}\left(r R_{K}\right)\right)}{2\left[K: K_{0}\right]} s_{2}(a)$ in Theorem $\left.2.8 \mathrm{~b}\right)$ in the form $\frac{\left.\operatorname{deg}_{2}\left(r R_{K}\right)\right)}{\left[K: K_{0}\right]} s_{2}^{\prime}(a)$ for some $s_{2}^{\prime}: K_{2 i-1}\left(R_{K}\right) \rightarrow H^{0}\left(K, \mathbf{Q}_{2} / \mathbf{Z}_{2}(i)\right)$. I am grateful to Pierre Colmez for pointing this out.

\section{Class field theory}

Let $F$ be again a finite extension of $\mathbf{Q}_{p}$. 
8.1. Theorem. For $i \geq 1$, the Dwyer-Friedlander spectral sequence yields isomorphisms

$$
\begin{aligned}
& K_{2 i-1}\left(F, \mathbf{Z} / l^{\nu}\right) \sim \\
& K_{2 i}\left(F, \mathbf{Z} / l^{\nu}\right) \stackrel{\sim}{\longrightarrow}\left(F, \mathbf{Z} / l^{\nu}(i)\right) \\
& H^{0}\left(F, \mathbf{Z} / l^{\nu}(i)\right) \oplus H^{2}\left(F, \mathbf{Z} / l^{\nu}(i+1)\right) .
\end{aligned}
$$

Proof. For $l \neq p$, this follows from [10, Th. 4.1]. For $l=p>2$, this follows from [9]. For $l=p=2$, this follows from e.g. [13, Th. 2] (using inter alia the Milnor conjecture) and the degeneracy of the Dwyer-Friedlander spectral sequence.

8.2. Corollary. For $i \geq 1$, we have a long exact sequence

$$
\begin{aligned}
0 \rightarrow H^{0}\left(F, \mathbf{Z} / l^{\nu}(i)\right) & \rightarrow K_{2 i-1}(F) \stackrel{l^{\nu}}{\rightarrow} K_{2 i-1}(F) \rightarrow H^{1}\left(F, \mathbf{Z} / l^{\nu}(i)\right) \\
& \rightarrow K_{2 i-2}(F) \stackrel{l^{\nu}}{\rightarrow} K_{2 i-2}(F) \rightarrow H^{2}\left(F, \mathbf{Z} / l^{\nu}(i)\right) \rightarrow 0 .
\end{aligned}
$$

Proof. This was proven in [10] under the assumption that Theorem 8.1 above holds ( $c f$. loc. cit., Remark 6.2). We give here an alternate proof.

First, the maps are defined in an obvious way from Theorem 8.1 by using the exact sequences

$$
\begin{gathered}
0 \rightarrow K_{2 i}(F) / l^{\nu} \rightarrow K_{2 i}\left(F, \mathbf{Z} / l^{\nu}\right) \rightarrow l^{\nu} K_{2 i-1}(F) \rightarrow 0 \\
0 \rightarrow K_{2 i-1}(F) / l^{\nu} \rightarrow K_{2 i-1}\left(F, \mathbf{Z} / l^{\nu}\right) \rightarrow l^{\nu} K_{2 i-2}(F) \rightarrow 0 \\
0 \rightarrow K_{2 i-2}(F) / l^{\nu} \rightarrow K_{2 i-2}\left(F, \mathbf{Z} / l^{\nu}\right) \rightarrow l^{\nu} K_{2 i-3}(F) \rightarrow 0 .
\end{gathered}
$$

Exactness is clear, except at the first, second, penultimate and ultimate terms. The composition

$$
K_{2 i}(F) / l^{\nu} \rightarrow K_{2 i}\left(F, \mathbf{Z} / l^{\nu}\right) \rightarrow H^{0}\left(F, \mathbf{Z} / l^{\nu}(i)\right)
$$

is 0 , as one sees by functoriality by passing to the algebraic closure $\bar{F}$ (by Suslin [25], $K_{2 i}(\bar{F}) / l^{\nu}=0$ ). This shows that the map $K_{2 i}(F) / l^{\nu} \rightarrow$ $H^{2}\left(F, \mathbf{Z} / l^{\nu}(i+1)\right)$ is injective, which in turn shows that the map $H^{0}\left(F, \mathbf{Z} / l^{\nu}(i)\right) \rightarrow{ }_{l^{\nu}} K_{2 i-1}(F)$ is injective.

It remains to see exactness at the second and ultimate terms. By a diagram chase, it is enough to prove it at the ultimate term. We shall use an $l$-adic argument as follows. Let $\hat{K}(F)$ be the $l$-completion of the $K$-theory spectrum $K(F)$. Then Theorem 8.1 implies that the natural map of spectra $\hat{K}(F) \rightarrow K^{\text {ét }}(F)$ induces an isomorphism on $\pi_{i}$ for all $i \geq 1$. In particular, for $i>1$,

$$
\hat{K}_{2 i-2}(F) \stackrel{\sim}{\longrightarrow} K_{2 i-2}^{\text {ét }}(F) \stackrel{\sim}{\longrightarrow} H^{2}\left(F, \mathbf{Z}_{l}(i)\right)
$$

where the second isomorphism comes as usual from the fact that $H^{0}\left(F, \mathbf{Z}_{l}(i-1)\right)=0$. 
In the Milnor exact sequence

$$
0 \rightarrow \lim ^{1} K_{2 i-1}\left(F, \mathbf{Z} / l^{\nu}\right) \rightarrow \hat{K}_{2 i-2}(F) \rightarrow \underset{\lim }{\longleftarrow} K_{2 i-2}\left(F, \mathbf{Z} / l^{\nu}\right) \rightarrow 0
$$

the left term vanishes because all terms in the $\lim ^{1}$ are finite by Theorem 8.1. The right term inserts into an exact sequence

$$
0 \rightarrow \lim _{\longleftarrow} K_{2 i-2}(F) / l^{\nu} \rightarrow \underset{\lim }{\longleftarrow} K_{2 i-2}\left(F, \mathbf{Z} / l^{\nu}\right) \rightarrow \underset{\lim _{l}}{l^{\nu}} K_{2 i-3}(F) .
$$

But $K_{2 i-2}\left(F, \mathbf{Q}_{l} / \mathbf{Z}_{l}\right) \simeq H^{0}\left(F, \mathbf{Q}_{l} / \mathbf{Z}_{l}(i-1)\right) \oplus H^{0}\left(F, \mathbf{Q}_{l} / \mathbf{Z}_{l}(i-1)\right)$ by Theorem 8.1 and Tate duality. Hence this group is finite and $K_{2 i-3}(F)\{l\}$ is finite as well. It follows that the right term of (8.1) vanishes. Summing up, we get an isomorphism

$$
\stackrel{\lim }{\longleftarrow} K_{2 i-2}(F) / l^{\nu} \stackrel{\sim}{\longrightarrow} H^{2}\left(F, \mathbf{Z}_{l}(i)\right)
$$

which is obviously compatible with the last map in the sequence of Corollary 8.2. It remains to observe that the map $H^{2}\left(F, \mathbf{Z}_{l}(i)\right) \rightarrow$ $H^{2}\left(F, \mathbf{Z} / l^{\nu}(i)\right)$ is surjective for all $\nu$. This completes the proof of Corollary 8.2 .

Let $a \in K_{2 i-1}(F)$. Assume that $l^{\nu}$ divides $\left|H^{0}\left(F, \mathbf{Q}_{l} / \mathbf{Z}_{l}(i)\right)\right|$. Then we have an isomorphism

$$
H^{1}\left(F, \mathbf{Z} / l^{\nu}(i)\right)=H^{1}\left(F, \mathbf{Z} / l^{\nu}\right) \otimes H^{0}\left(F, \mathbf{Z} / l^{\nu}(i)\right)
$$

hence the homomorphism of Corollary 8.2 carries $a$ to an element of the form $\chi_{a} \otimes x$, where $\chi_{a} \in H^{1}\left(F, \mathbf{Z} / l^{\nu}\right)$ and $x$ is a generator of $H^{0}\left(F, \mathbf{Z} / l^{\nu}(i)\right)$. The cyclic extension $E=F\left(\chi_{a}\right)$ does not depend on the choice of $\chi_{a}$ and $x$. Corollary 8.2 implies that $a_{E}$ is of the form $l^{\nu} b$ for some $b \in K_{2 i-1}(E)$. Conversely, if $L$ is an extension of $F$ such that $a_{L}$ is divisible by $l^{\nu}$, then $\chi_{a}$ vanishes on $L$, hence $L \supseteq E$. This justifies the notation $E=F\left(\frac{1}{l^{\nu}} a\right)$.

Let $G=\operatorname{Gal}(E / F)$ : this is a cyclic group of order dividing $l^{\nu}$. Let $g \in G$ and $b$ be as above. Then $g b-b \in{ }_{l^{\nu}} K_{2 i-1}(E) \simeq H^{0}\left(E, \mathbf{Z} / l^{\nu}(i)\right)$, still by Corollary 8.2. Hence we get a 1-cocycle of $G$ with values in $H^{0}\left(E, \mathbf{Z} / l^{\nu}(i)\right)$, or an element of $H^{1}\left(G, H^{0}\left(E, \mathbf{Z} / l^{\nu}(i)\right)\right)$. Pushing this element into $H^{1}\left(F, \mathbf{Z} / l^{\nu}(i)\right)$, we get back the image of $a$ under the map of Corollary 8.2: this is obvious.

Note that, in fact, $G$ acts trivially on $H^{0}\left(E, \mathbf{Z} / l^{\nu}(i)\right)$ because this group coincides with $H^{0}\left(F, \mathbf{Z} / l^{\nu}(i)\right)$. Hence we have defined above a homomorphism from $G$ to $H^{0}\left(F, \mathbf{Z} / l^{\nu}(i)\right)$, or a homomorphism

$$
\psi_{a}: \operatorname{Gal}(\bar{F} / F)^{a b} \rightarrow H^{0}\left(F, \mathbf{Z} / l^{\nu}(i)\right)=\mathbf{Z} / l^{\nu}(i) .
$$

We now choose $l^{\nu}=\left|H^{0}\left(F, \mathbf{Q}_{l} / \mathbf{Z}_{l}(i)\right)\right|$. Let $f \in F^{*}$. Denote by $\operatorname{Art}(f)$ its Artin symbol in $\operatorname{Gal}(\bar{F} / F)^{a b}$. 
8.3. Theorem. We have

$$
(a, f)=\psi_{a}(\operatorname{Art}(f)) .
$$

Proof. If $\chi \in H^{1}\left(F, \mathbf{Z} / l^{\nu}\right)$, then we have by [20, ch. XIV, p. 212, prop. 3]

$$
\chi(\operatorname{Art}(f))=\operatorname{inv}(f \cdot \delta(\chi))
$$

where $f$ is viewed as an element of $H^{0}\left(F, \mathbb{G}_{m}\right)$ and $\delta$ is the Bockstein from the exact sequence $0 \rightarrow \mathbf{Z} \rightarrow \mathbf{Z} \rightarrow \mathbf{Z} / l^{\nu} \rightarrow 0$. On the other hand, an easy cocycle computation (which strangely is not in [20], but see loc. cit., ch. XIV, p. 214, proof of prop. 5) shows that

$$
f \cdot \delta(\chi)=\chi \cdot(f)
$$

where $(f)$ is the Kummer class of $f$ in $H^{1}\left(F, \mathbf{Z} / l^{\nu}(1)\right)$.

Twisting this computation by $\mathbf{Z} / l^{\nu}(i)$, we get

$$
\psi_{a}(\operatorname{Art}(f))=\theta_{l^{\nu}}^{-1}\left(\psi_{a} \cdot(f)\right)
$$

where $\theta_{l^{\nu}}$ is the isomorphism of (3.2) (use Remark 3.3).

On the other hand,

$$
(a, f)=h^{(i)}(a \cdot\{f\})=\theta_{l^{\nu}}^{-1}\left(\operatorname{ch}_{i+1}(a \cdot\{f\})\right.
$$

where $c h_{i+1}$ is the Dwyer-Friedlander map of (4.2). By the multiplicativity of the Dwyer-Friedlander spectral sequence, we have

$$
\operatorname{ch}_{i+1}(a \cdot\{f\})=\operatorname{ch}_{i}(a) \cdot \operatorname{ch}_{1}(\{f\})=c h_{i}(a) \cdot(f)
$$

where $c_{i}: K_{2 i-1}(F) \rightarrow H^{1}\left(F, \mathbf{Z} / l^{\nu}(i)\right)$ is the edge homomorphism stemming from this spectral sequence. It remains to see that

$$
\psi_{a}=\operatorname{ch}_{i}(a)
$$

which follows tautologically from the definition of the map $K_{2 i-1}(F) \rightarrow$ $H^{1}\left(F, \mathbf{Z} / l^{\nu}(i)\right)$ in Corollary 8.2.

\section{REFERENCES}

[1] D. Arlettaz, G. Banaszak On the non-torsion elements in the algebraic $K$-theory of rings of integers, J. Reine Angew. Math. 461 (1995), 63-79.

[2] G. Banaszak Generalization of the Moore exact sequence and the wild kernel for higher K-groups, Compositio Math. 86 (1993), 281-305.

[3] S. Bloch Lectures on algebraic cycles, Duke Univ. Math. Series IV, 1980.

[4] W.J Cassels, A. Fröhlich Algebraic Number Theory, Acad. Press, 1967.

[5] W.G. Dwyer, E.M. Friedlander Algebraic and étale K-theory, Trans. Amer. Math. Soc. 292 (1985), 247-280.

[6] W. G. Dwyer, E. M. Friedlander, S. A. Mitchell, The generalised Burnside ring and the K-theory of ring with roots of unity, K-theory 6 (1992), 285-300.

[7] H. Hamraoui Un facteur direct canonique de la $K$-théorie d'anneaux d'entiers algébriques non exceptionnels, C. R. Acad. Sci. Paris 332 (2001), 957-962. 
[8] B. Harris, G. Segal, $K_{i}$-groups of rings of algebraic integers, Annals of Math. 101 (1975), 20-33.

[9] L. Hesselholt, I. Madsen On the K-theory of local fields, to appear in the Ann. of Math.

[10] B. Kahn On the Lichtenbaum-Quillen conjecture, Algebraic $K$-theory and algebraic topology, NATO ASI Series, Ser. C. 407 (1993), 147-166.

[11] B. Kahn Bott elements in algebraic K-theory, Topology 35 (1997), 963-1006.

[12] B. Kahn The Quillen-Lichtenbaum conjecture at the prime 2, preprint, 1997.

[13] B. Kahn K-theory of semi-local rings with finite coefficients and étale cohomology, K-theory 25 (2002), 99-139.

[14] B. Kahn Équivalences rationnelle et numérique sur certaines variétés de type abélien sur un corps fini, to appear in the Ann. Sci. Ec. Norm. Sup.

[15] K. Kato and S. Saito Unramified class field theory of arithmetical surfaces, Ann. of Math. 118 (1983), 241-275.

[16] J.S. Milne Arithmetic duality theorems, Perspectives in Math., Acad. Press, 1986.

[17] C.C Moore Group extensions of p-adic and adelic linear groups, Publ. Math. IHES 35, 1971.

[18] J. Milnor Introduction to algebraic K-theory, Ann. of Math. Studies 72, Princeton Univ. Press, 1971.

[19] D. Quillen On the cohomology and K-theory of the general linear group over a finite field, Ann. of Math. 96 (1972), 552-586.

[20] J.-P. Serre Corps locaux (2nd edition), Hermann, 1968.

[21] J.-P. Serre Cohomologie galoisienne (5th edition), Lect. Notes in Math. 5, Springer, 1994.

[22] J. Rognes, C. Weibel Étale descent for two-primary algebraic K-theory of totally imaginary number fields, K-Theory 16 (1999), 101-104.

[23] C. Soulé Groupes de Chow et $K$-théorie de variétés sur un corps fini, Math. Ann. 268 (1984), 317-345.

[24] A.A. Suslin On the K-theory of algebraically closed fields, Invent. Math. 73 (1983), 241-245.

[25] A.A. Suslin On the K-theory of local fields, J. Pure Appl. Algebra 34 (1984), 301-318.

Institut de Mathématiques de Jussieu, 175-179 Rue du Chevaleret, 75013 PARIS, France

E-mail address: kahn@math.jussieu.fr 